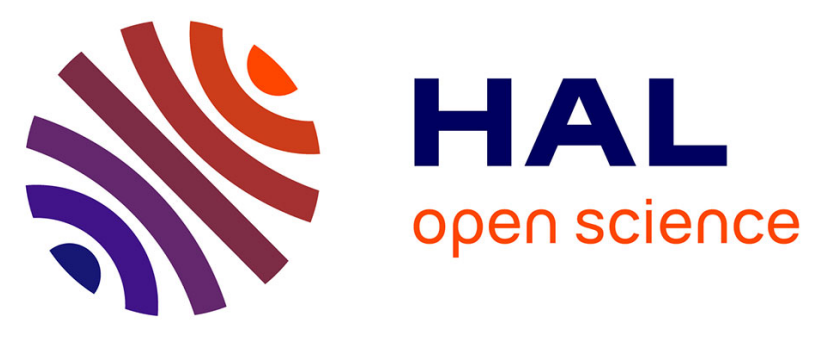

\title{
Diversité des pratiques funéraires et espaces sépulcraux sectorisés au Chasséen ancien sur le site du Crès à Béziers (Hérault) : croisements de données archéologiques et anthropologiques \\ Gilles Loison, Aurore Schmitt
}

\section{To cite this version:}

Gilles Loison, Aurore Schmitt. Diversité des pratiques funéraires et espaces sépulcraux sectorisés au Chasséen ancien sur le site du Crès à Béziers (Hérault): croisements de données archéologiques et anthropologiques. Gallia Préhistoire - Préhistoire de la France dans son contexte européen, 2009, 51, pp.245-272. 10.3406/galip.2009.2480 . halshs-01896935

\section{HAL Id: halshs-01896935 \\ https://shs.hal.science/halshs-01896935}

Submitted on 19 Dec 2019

HAL is a multi-disciplinary open access archive for the deposit and dissemination of scientific research documents, whether they are published or not. The documents may come from teaching and research institutions in France or abroad, or from public or private research centers.
L'archive ouverte pluridisciplinaire HAL, est destinée au dépôt et à la diffusion de documents scientifiques de niveau recherche, publiés ou non, émanant des établissements d'enseignement et de recherche français ou étrangers, des laboratoires publics ou privés.

\section{(ㅇ)(1) $\$$}

Distributed under a Creative Commons Attribution - NonCommercial - NoDerivatives 44.0 


\title{
DIVERSITÉ DES PRATIQUES FUNÉRAIRES ET ESPACES SÉPULCRAUX SECTORISÉS AU CHASSÉEN ANCIEN SUR LE SITE DU CRÈS À BÉZIERS (HÉRAULT) Croisements de données archéologiques et anthropologiques
}

\author{
Gilles LOISON* et Aurore SCHMITT**
}

\begin{abstract}
Mots-clés. Chasséen ancien, habitat, sépultures, pratiques funéraires.
Résumé. Cet article a pour but de présenter les premiers résultats des travaux engagés sur le site chasséen ancien du Crès à Béziers, fouillé dans le cadre d'une opération d'archéologie préventive. Sur 190 structures excavées découvertes, 33 ont été le réceptacle de dépôts funéraires, représentant une population de défunts de 49 individus. C'est à ce jour le plus gros corpus funéraire pour cette période dans le sud de la France. Cette forte composante funéraire, qui par ailleurs présentait une forte variabilité et une grande complexité d'organisation, pouvait être susceptible de répondre aux questions récurrentes qui concernent les pratiques funéraires de cette étape constitutive du Chasséen méridional.

Nous avons donc cherché à comprendre l'ordonnancement de cette population inhumée et sa représentativité en confrontant les données archéologiques et celles de l'anthropologie. Ce premier niveau d'interprétation a permis d'illustrer la question des tombes en milieu d'habitat et de montrer l'extrême diversité des modes funéraires qui caractérise les inhumations chasséennes, ici contemporaines et présentes en même lieu. Cette apparente dichotomie entre les inhumations en milieu d'habitat et les tombes architecturées constituées en un petit cimetière est tout à fait remarquable. Elle peut être la résonance d'une société stratifiée, où les individus peuvent avoir sans distinction d'âge et de sexe des statuts distincts et probablement hiérarchisés.
\end{abstract}

Keys-words. Early Chassean, habitat, burial, funerary practices.

Abstract. The goal of this article is to present the first results of research conducted on the Early Chassean site of Crès à Béziers, which was excavated in the context of a preventive operation. Out of the 190 excavated structures discovered, 33 served as receptacles for funerary deposits, representing a population of 49 deceased individuals. This is currently the largest funerary ensemble known for this period in southern France. This strong funerary component, which also shows significant variability and organizational complexity, could respond to recurring questions concerning the funerary practices of this stage of the southern Chassean.

In our work, we thus attempted to understand the sequencing of this inhumed population and its representivity through a confrontation of archaeological and physical anthropological data. This first level of interpretation allowed us to address the question of tombs in a habitat context and to show the extreme diversity of the funerary practices that characterize Chassean inhumations, here contemporary and present in the same location. This apparent dichotomy between inhumations in habitat contexts and architectural tombs constituted in a small cemetery is quite remarkable. It could be the resonance of a stratified society in which individuals, regardless of their age or sex, can have distinct and probably hierarchical statuses.

Translation: Magen O'FARRELL

\footnotetext{
* Institut National de Recherches Archéologiques Préventives et UMR 5608 TRACES, CRPPM, 39, allées Jules Guesde, 31100 Toulouse. Courriel: gilles.loison@inrap.fr

** CNRS - UMR 6578, Anthropologie bio-culturelle, Adaptabilité biologique et culturelle, Faculté de Médecine Nord, Boulevard Pierre Dramard, 13916 Marseille Cedex 20. Tel: 0496152724 - Fax: 04916080 62. Courriel: aurore.schmitt@univmed.fr
} 
Schlüsselwörter. Frühes Chasséen, Siedlung, Grabstätten, Bestattungssitten.

Zusammenfassung. Ziel dieses Artikels ist es, die ersten Erkenntnisse vorzustellen, die im Rahmen einer Präventivgrabung auf dem in das frühe Chasséen datierten Fundplatz Le Crès in Béziers gewonnen wurden. Von 190 ergrabenen Strukturen enthielten 33 Grabbeigaben; sie entsprechen 49 bestatteten Individuen. Bis heute ist dies das umfangreichste Corpus von Bestattungen aus dieser Periode in Südfrankreich. Der hohe Anteil an Bestattungen, die darüber hinaus eine hohe Variabilität und eine große organisatorische Komplexität aufweisen konnten, ist geeignet, die wiederholt auftretenden Fragen bezüglich der Bestattungssitten dieser grundlegenden Etappe, in der sich das Chasséen Méridional herausgebildet hat, zu beantworten.

Wir haben daher versucht, das Organisationssystem der Anordnung der bestatteten Population und deren repräsentativen Charakter zu verstehen, indem wir die archäologischen und die anthropologischen Daten einander gegenübergestellt haben. Dieser erste Interpretationsansatz hat es ermöglicht, die Problematik der Bestattungen im Siedlungsbereich zu veranschaulichen und die extreme Vielfalt der Bestattungsarten aufzuzeigen, die für diese aus der gleichen Zeit und von der gleichen Fundstelle stammenden Bestattungen des Chasséen bezeichnend ist. Diese offensichtliche Dichotomie zwischen den Bestattungen im Siedlungsbereich und den baulich gestalteten, in einer kleinen Nekropole zusammengefassten Gräbern ist durchaus bemerkenswert. Sie spiegelt möglicherweise eine geschichtete Gesellschaftsstruktur wider, in der die Individuen ohne Unterscheidung von Alter und Geschlecht unterschiedliche und wahrscheinlich hierarchisierte Stellungen einnehmen.

Übersetzung: Isa ODENHARDT-DONVEZ

\section{PRÉSENTATION GÉNÉRALE}

Le point de départ de cet article a été le souhait de publier les premiers résultats de l'étude anthropo-biologique de la population du site chasséen du Crès à Béziers. Cette étude a été reprise et développée récemment par A. Schmitt, grâce aux moyens apportés par l'ACR Espaces et expressions funéraires au Néolithique moyen dans le monde pyrénéen et ses marges coordonnée par J. Vaquer. Ces résultats très attendus, auxquels s'ajoutaient ceux des datations radiocarbone, rendaient possible l'approche synthétique de l'organisation du site. Il est nous apparu nécessaire, avant l'achèvement de la monographie, de présenter ces premiers résultats afin, d'une part, de répondre à l'impatience de nos collègues et, d'autre part, de clarifier certaines interprétations hâtives faisant référence à ce site.

Fouillé en 2001, à l'occasion des travaux routiers du contournement nord de Béziers, le gisement du Crès est situé à $4 \mathrm{~km}$ au nord-ouest de cette agglomération (Loison et al., 2003 et 2004). Proche de l'Orb, celui-ci s'inscrit sur un paléochenal déjà colmaté à la fin du Pléistocène, parallèle au cours actuel. Hormis quelques témoins ténus d'occupations du Paléolithique supérieur et du Néolithique final, la période la plus densément représentée est celle qui correspond au Néolithique moyen 2, et plus précisément à la phase ancienne du Chasséen méridional. Douze datations radiométriques ont donné une fourchette probabiliste maximale d'occupation comprise entre de 4350 à 4000 av. J.-C., confirmant les résultats des études chronotypologiques du mobilier (Jedikian, 2004; Léa, 2004).
Ce gisement a été étudié sur une surface d'environ $5000 \mathrm{~m}^{2}$, qui correspondait à la partie du site menacée par les travaux. Le faible enfouissement des vestiges n'a pas permis la préservation des sols d'occupation, et l'érosion a été estimée selon les secteurs à $30 \mathrm{~cm}$ ou $40 \mathrm{~cm}$.

L'occupation chasséenne est matérialisée par 190 structures en fosses qui se cantonnent quasi exclusivement dans l'emprise du chenal fossile, indiquant un développement longitudinal de l'occupation. Seules ses limites est et ouest ont pu être cernées. Toutefois, des fragments de meules, ramassés lors de prospections dans les parcelles limitrophes situées au nord de la fouille (les seules qui étaient accessibles), laissent indiquer que l'occupation chasséenne serait effectivement plus étendue. Il est donc important de souligner que, même si la surface d'investigation est importante, il ne s'agit que d'un échantillon aléatoire de ce site, biais qui n'est pas sans conséquence pour l'interprétation de son organisation.

La forte composante funéraire de ce site d'habitat décelée dès l'évaluation, et un temps d'intervention limité, ont conduit à privilégier, durant la fouille, l'approche archéo-anthropologique. Les faits nous ont donné raison puisque cette partie du site a livré les restes de 49 individus plus ou moins bien représentés au sein de 30 structures funéraires. Il s'agit à ce jour du plus important ensemble de structures funéraires et d'individus chasséens étudiés pour le midi de la France.

Les résultats récents de l'étude anthropo-biologique ont permis de rediscuter des modalités de constitution de cet ensemble et, en confrontant ces données à celles de l'étude 
archéologique (Loison et al., 2004), d'approfondir l'examen de l'organisation de la population inhumée: distribution spatiale des structures funéraires, présence des dépôts pluriels, chronologie interne des dépôts.

Rappelons qu'une population d'inhumés ne correspond que très rarement à la totalité de la population décédée, notamment au Néolithique moyen (Duday, 1987; Loison, 1998; Chambon, Leclerc, 2007; Vaquer et al., 2008); il nous semblait donc pertinent de distinguer, à partir des paramètres disponibles - outre le fait que le site n'a pas été totalement fouillé -, l'origine des biais qui ont pu conduire à une telle composition de la population et, au-delà, de nous interroger sur sa représentativité.

\section{LES DONNÉES ARCHÉOLOGIQUES}

\section{LES DIFFÉRENTES STRUGTURES}

Une présentation détaillée de ces témoins est nécessaire à la compréhension de l'exposé. Les 190 structures correspondant à cette occupation chasséenne ont des morphologies très variables, elles possèdent toutefois très majoritairement une ouverture circulaire. Une grande majorité d'entre elles possèdent dans leur comblement des rejets domestiques plus ou moins importants, témoins d'une proximité d'habitats. Quelques-unes contiennent parfois des dépôts funéraires individuels ou pluriels. Seules 11 d'entre elles ont une forme oblongue et se rapportent à des fosses spécifiquement funéraires. Les diamètres à l'ouverture vont de $0,25 \mathrm{~m}$ à 2,70 $\mathrm{m}$ et leurs profondeurs conservées de $0,10 \mathrm{~m}$ à $1,40 \mathrm{~m}$, pour des volumes variant de $0,22 \mathrm{~m}^{3}$ à $7,70 \mathrm{~m}^{3}$. Quatre types principaux de structures domestiques se distinguent: les fosses de forme tronconique et piriforme de type silo, les fosses emboîtées en sablier, les fosses de grand diamètre profondes, et les fosses de grand diamètre peu profondes (fig. 1).

\section{LES FOSSES DE FORME TRONCONIQUE OU PIRIFORME DE TYPE SILO (SR)}

Elles possèdent un diamètre d'embouchure plus réduit que le fond (0,80 m à $1 \mathrm{~m}$ pour l'ouverture et jusqu'à 2,10 m pour le fond).

Ces fosses sont au nombre de 33. Elles sont majoritairement situées dans la moitié nord de la zone fouillée et s'inscrivent dans trois concentrations principales. La première est située au nord-ouest, elle borde une aire de forme elliptique dépourvue d'excavation. Parmi elles, 3 fosses comportaient des dépôts funéraires simples et pluriels. La deuxième concentration se situe plus au sud, où il semble qu'elles n'ont eu qu'un usage domestique. La troisième concentration est présente dans la partie sud-est, 2 de ces fosses ont accueilli des sépultures humaines ainsi que des dépôts de chiens.

En raison de leur forme, elles sont assez systématiquement apparentées à des structures d'ensilage, sans autre caractère démonstratif induisant le fait qu'elles ont contenu des céréales. Lors de la fouille, nous avons cherché des indices permettant de valider ou non l'hypothèse du silo à céréales. Si des grains de céréales carbonisées se trouvaient bien au sein des remplissages, ceux-ci se situaient non pas en concentration sur le fond, mais inclus dans les sédiments de comblement, en position secondaire, et de plus en très faible quantité. Nous avons également effectué une étude paléo-entomologique pour une recherche d'insectes ravageurs des céréales (travaux Estelle Olive, inédits), mais, faute d'un milieu favorable à leur conservation, celle-ci n'a donné aucun résultat. Nous espérons que l'étude micromorphologique en cours pourra nous apporter des informations complémentaires (travaux Julia Wattez, inédits). Le seul indice indirect, qui permet d'aller dans ce sens, est la mise en évidence dans certaines fosses d'une couche homogène de terre malaxée appliquée sur les parois, formant une sorte de «chemisage». Nous pensons que ce dispositif, s'il est validé par l'étude micromorphologie, a pu servir à créer un milieu anaérobie favorable à la conservation de denrées végétales.

Soulignons que, sur ce site, l'usage de ce type de fosse semble bien lié à des activités domestiques: sur les 33 fosses dénombrées, seules 7 ont servi de structure d'accueil à des défunts humains et canins.

\section{LES FOSSES EMBOÎTÉES EN SABLIER (SAB)}

Au nombre de 5, elles sont concentrées dans la partie nord-ouest. La partie inférieure de ces aménagements correspond morphologiquement à celle d'un silo, mais son volume est plus réduit. Leur usage est exclusivement de nature domestique.

\section{LES FOSSES DE GRAND DIAMÈTRE PROFONDES (GDP)}

Elles sont également au nombre de 5: 2 se situent au nord-ouest au sein d'une concentration de 4 fosses de type silo. Ce sont les fosses les plus volumineuses: $7,50 \mathrm{~m}^{3}$ pour la première et $5,80 \mathrm{~m}^{3}$ pour la seconde. Ces structures de 


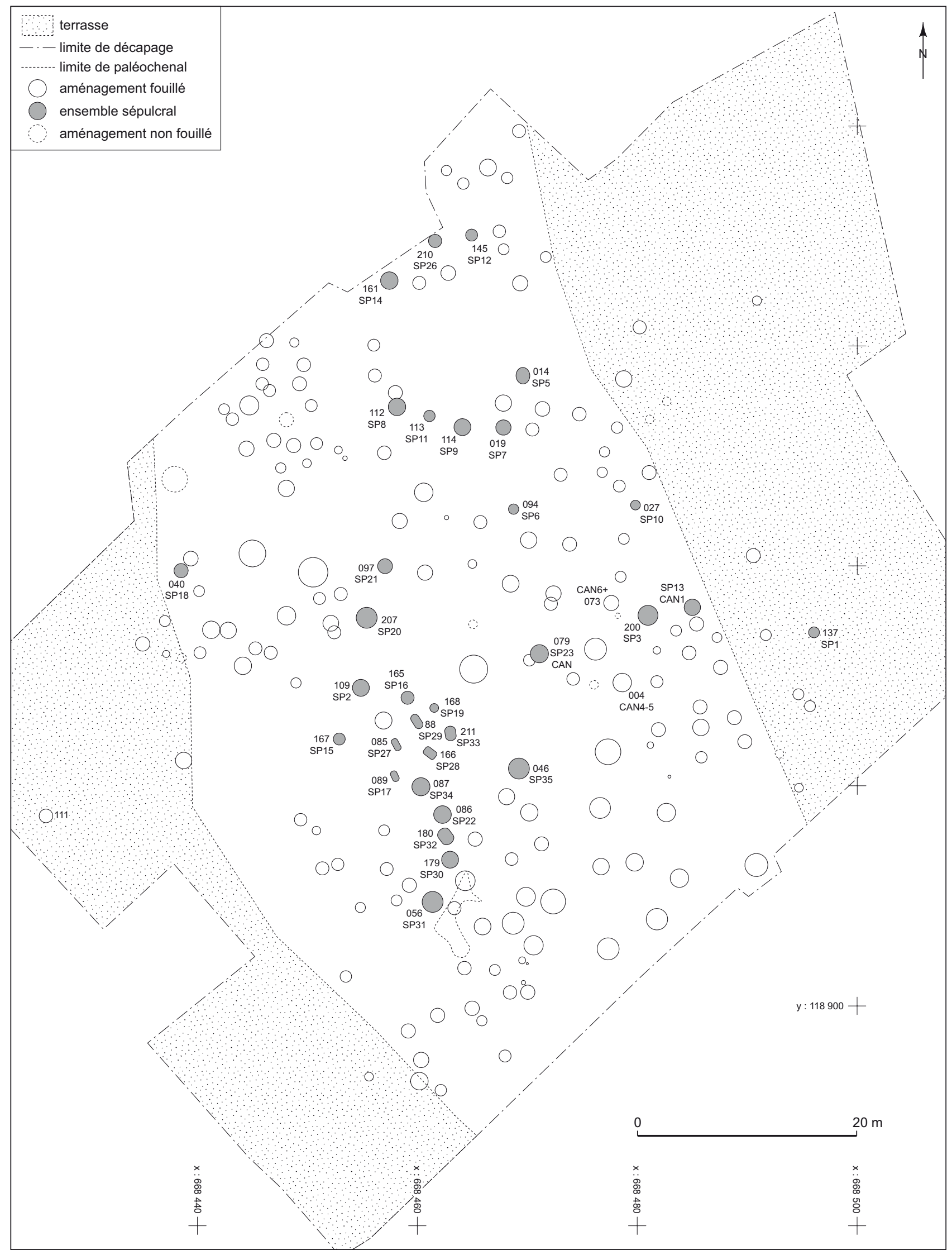

Fig. 1 - Situation des différentes structures (DAO: F. Audouit, C. Bioul, C. Labarussiat). 
forme cylindrique à fond plat, dont le diamètre varie entre 2,10 $\mathrm{m}$ et 2,60 $\mathrm{m}$ et la profondeur entre $0,70 \mathrm{~m}$ et 2,60 $\mathrm{m}$, ont pu avoir un usage comparable à celui d'une cave. On note, de plus, un probable lien de proximité entre elles et les fosses de type silo.

Précisons qu'une fosse de ce type est située près d'une aire de forme elliptique dépourvue d'excavation (au nordest) : elle est la structure d'accueil de sépultures plurielles (Amt 112 SP 8).

\section{LES FOSSES DE GRAND DIAMÈTRE PEU PROFONDES (GDPP)}

Elles sont assez nombreuses : 16 au total, plutôt présentes dans la partie sud de la zone fouillée. Elles présentent un diamètre qui peut aller de $1,60 \mathrm{~m}$ à 2,40 m, pour une profondeur variant de $0,20 \mathrm{~m}$ à $0,40 \mathrm{~m}$. Leur comblement est variable, il comporte pour certaines des rejets domestiques de proximité.

Parmi toutes les fosses de ce type, seules 4 renfermaient des dépôts humains (Amt 46, 86, 109, 114). L'une associait une inhumation humaine et le dépôt d'un chien (Amt 7), une autre comportait les dépôts exclusifs de 2 chiens (Amt 73). Soulignons que ce type de structure, comme les fosses de type silo, présente plus de cas d'un usage domestique que funéraire. Les GDPP semblent donc elles aussi faire l'objet d'un remploi pour cette fonction ultime.

\section{APPROCHES GÉNÉRALES SUR L'ORGANISATION SPATIALE}

La distribution de chacun de ces types de structure est variable et permet d'apporter des indications sur l'organisation de l'espace occupé. Ces associations typologiques et des spécificités de remplissages et de dépôts permettent d'isoler 6 assemblages distincts (fig. 2). Certains de ces groupements sont assez évidents (groupes 1, 2 et 6), attestant une sectorisation de l'espace occupé, d'autres apparaissent plus hypothétiques (groupes 3, 4 et 5).

Le groupe 1, au nord-ouest, est caractérisé par une forte concentration de structures excavées, principalement constituée de fosses de type silo (SR) et de fosses en sablier (SAB). Leur comblement présente un sédiment nettement moins anthropisé et détritique que celui des autres groupes, indiquant un relatif éloignement de l'espace domestique. Il pourrait s'agir d'une aire dévolue au stockage.

Le groupe 2 est situé à l'est du premier. Les fosses forment une couronne délimitant un espace dépourvu d'excavation, dont la surface est d'environ $200 \mathrm{~m}^{2}$. Cette délimitation artificielle pourrait correspondre à l'emplacement d'une construction dont les traces au sol auraient disparu. Une configuration similaire a été observée sur le site du Pirou à Valros fouillé récemment (Loison et Gleyze, à paraître). Parmi les 6 fosses de type silo qui s'y rattachent, 3 comportaient les restes de un et plusieurs individus (SP 5, SP 26 et SP 12). Une fosse de type GDP, ainsi qu'une autre de type GDPP, s'inscrivent également dans ce groupe; elles sont toutes deux le siège de dépôts funéraires. Pour la première, il s'agit de dépôts pluriels (SP 8) et, pour la seconde, d'une inhumation individuelle (SP 9). Soulignons que, dans ce groupe, 8 excavations renferment des défunts.

Nous ne détaillerons pas ici les groupes 3 et 5, qui ne présentent pas d'intérêt particulier pour notre propos.

Le groupe 4, situé dans le quart sud-est de la zone étudiée, forme une petite concentration constituée de 4 fosses de type silo (Amt 6, 34, 79 et 200), dont 2 ont été utilisées en espace funéraire (SP 3 et SP 23), ainsi que de 4 fosses de type GDPP, dont 2 employées à des fins funéraires, et de 2 fosses de type GDP. Toutefois, la spécificité de ce groupe est la présence de 6 inhumations de chiens, au sein de fosse de type silo ou de type GDPP (Amt 73 et Amt 4), en dépôts simples et pluriels (déterminations V. Forest). Certains de ces derniers ont d'ailleurs bénéficié des mêmes pratiques que les humains (emballage, espace vide). Cependant, dans ce groupe, il existe également un cas associant, dans la même fosse de type GDPP, un dépôt de chien à celui d'un humain (SP 13). Dans ce cas, la question de «morts d'accompagnement» peut être envisagée (l'expression est due à A. Testart, 2004). De plus, dans le comblement d'une fosse de type silo contenant 2 dépôts, probablement successifs, de 2 chiens, ont été mis au jour 2 ossements humains se rapportant à 2 individus distincts (un fragment de calotte crânienne et une prémolaire). Si, pour l'élément crânien, on ne peut exclure la question d'un dépôt volontaire, car 2 autres cas d'os isolés ont été découverts dans les ensembles SP 12 et SP 3 (extrémités de fémurs), il existe une forte probabilité pour que la dent soit en position erratique.

Les dépôts de chiens en contexte funéraire et d'habitat sont une composante culturelle du Chasséen méridional. Ils ont notamment été reconnus en association avec des dépôts humains sur les sites de la Batisdonne à Trets dans les Bouches-du-Rhône (Courtin, 1974), dans la fosse 6 des Martins à Roussillon dans le Vaucluse (D'Anna, 1993), et dans la fosse 70 de Saint-Paul-Trois-Châteaux dans la Drôme (Beeching, Crubézy, 1998, p. 153), mais des dépôts exclusifs de chiens ont également été découverts sur le site d'habitat 


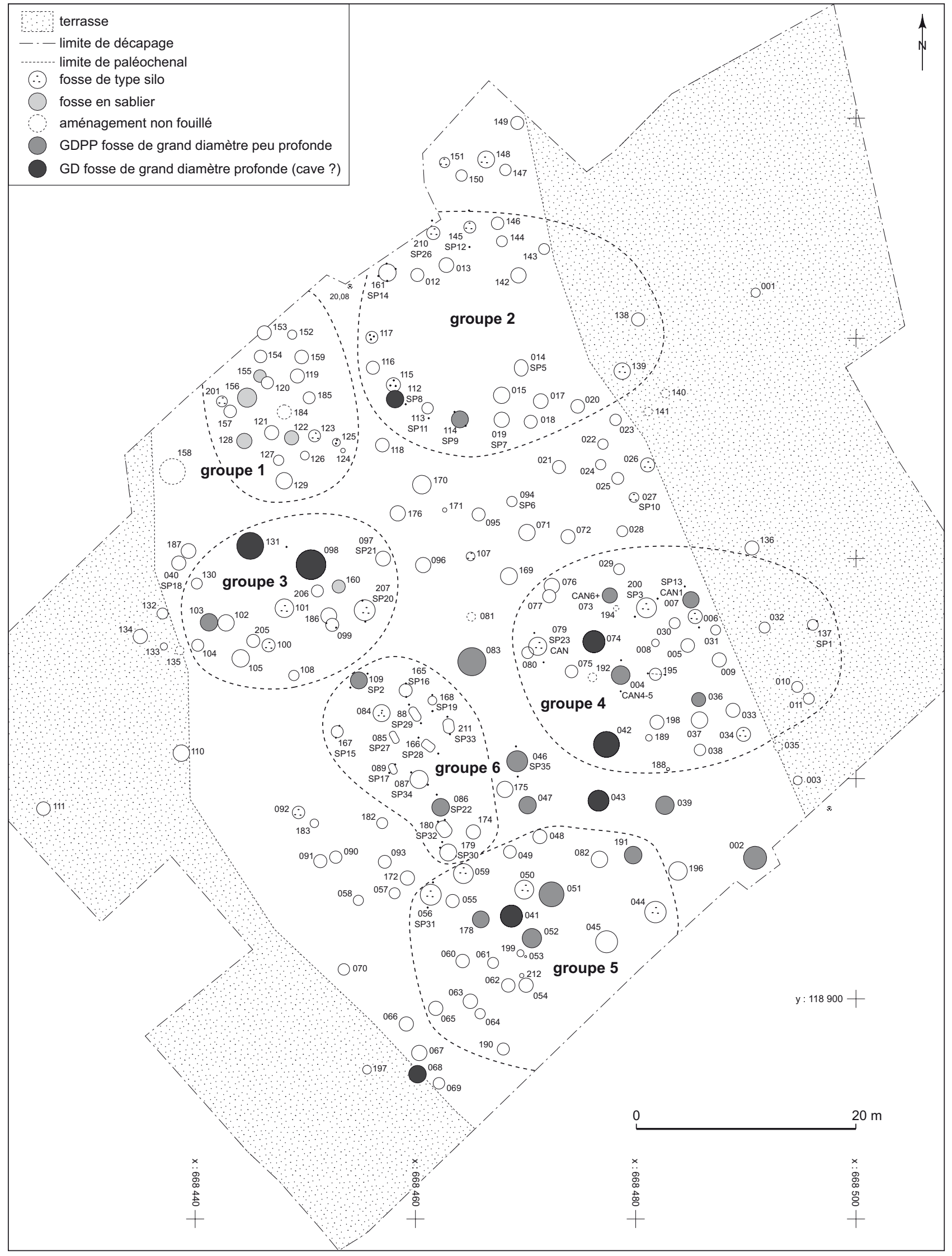

Fig. 2 - Organisation spatiale des différents types de structures (DAO: F. Audouit, C. Bioul, C. Labarussiat). 
chasséen ancien des Plots à Berriac dans l'Aude (Vaquer, 1998). Toutefois, le fait nouveau est que, sur le site du Crès, il est établi que certains de ces animaux ont bénéficié d'un traitement funéraire semblable à celui destiné aux défunts humains.

Le groupe 6 est situé au centre de la zone étudiée, entre les groupes 3, 4 et 5 . Il est constitué quasi exclusivement de fosses sépulcrales, dont la concentration en forme de fuseau se développe selon un axe nord-sud. Les aménagements funéraires rencontrés dans ce groupe sont typologiquement distants des autres ensembles. Ce sont pour la plupart des fosses de forme oblongue simple ou présentant un aménagement interne pour un accès latéral, de type à cavité latérale ou en niche.

Il s'agit manifestement d'un espace funéraire à part entière, isolé des espaces domestiques, qui a été préservé de tout autre type de creusement. Celui-ci est si bien imbriqué dans l'espace général, qu'il ne peut chronologiquement être postérieur aux autres groupes. Sa contemporanéité relative avec ces derniers, également détenteur de tombes (cf. infra, Chronologie absolue et organisation spatiale des structures funéraires), pose la question de la coexistence en un même espace de pratiques funéraires apparemment aussi différentes.

\section{LES STRUCTURES FUNÉRAIRES}

Sur les 190 aménagements reconnus, 30 recelaient des inhumations, individuelles et plurielles, correspondant à 49 individus, présences exceptionnelles pour un site de cette période. Ces ensembles sépulcraux sont d'une étonnante diversité et d'une grande complexité, aussi bien dans l'agencement des dépôts que dans le mode de traitement des corps. Toutefois, deux grandes catégories de tombes se dégagent (fig. 3) : les fosses sépulcrales, excavations confectionnées à des fins exclusivement funéraires, et les structures morphologiquement similaires aux fosses domestiques, qui ont selon toute vraisemblance été réutilisées en espace funéraire.

\section{LES FOSSES ÉLABORÉES À DES FINS SPÉCIFIQUEMENT FUNÉRAIRES}

Elles se déclinent en deux types principaux: la fosse sépulcrale simple et la fosse sépulcrale à cavité latérale.

- La fosse sépulcrale simple (FS) est de forme oblongue ou plus ou moins ronde et peu profonde, et ne comporte qu'un seul individu dont la décomposition se serait déroulée en espace colmaté, indiquant une inhumation en pleine terre. Elle est représentée par 6 unités: SP 1, SP 4, SP 6, SP 15, SP 28 et probablement SP 19, situées relativement en marge des concentrations décrites. L'orientation des corps est majoritairement sud-nord.

- La fosse sépulcrale à cavité latérale (FSOB). Il s'agit de cavités creusées en forme de niche et comportant un accès latéral. Elles présentent cependant quelques variantes architecturales: la structure peut être simple (SP 27, SP 29, SP 32, SP 33) ou comporte parfois deux espaces distincts, la cavité latérale s'ouvrant dans la paroi d'une fosse plus grande, probable structure d'accès (SP 22 et SP 30). Cette cavité, après le dépôt du corps, était fermée latéralement par plusieurs dalles de calcaire posées de chant en position plus ou moins oblique, la partie supérieure prenant appui sur le bord supérieur de l'excavation. Toutefois, la plupart de ces cavités, parmi les plus simples, ont eu leur partie supérieure tronquée par les travaux agricoles, faisant disparaître la voûte. Au décapage, elles sont apparues sous la forme d'un demi-cercle ou d'un haricot, mais elles ont généralement pu être distinguées sans difficulté en raison de la présence des blocs de calcaire disposés de chant, vestiges de leur dispositif latéral de fermeture.

Ces fosses, dont l'axe longitudinal est généralement nord-sud, contiennent un seul individu dont la décomposition se serait réalisée en espace vide. Les corps sont orientés sud-nord. Ces structures funéraires sont exclusivement présentes dans le groupe 6 .

La tombe la plus élaborée de cette catégorie (SP 22) (fig. 4 et 5) présente un surcreusement pratiqué dans la paroi est d'une fosse formant une véritable niche. Cette cavité a servi de réceptacle à un défunt, puis elle a été soigneusement fermée par des dalles disposées selon un plan incliné. Ce dispositif incluait une meule complète et sa molette. Ce type d'architecture particulier, que l'on retrouve partiellement dans la SP 30, présenterait des similitudes avec certaines tombes de la culture catalane des sepulcros de fosa (Bosch, Faura, 2003; Loison et al., 2004; Labriffe et al., 2007).

\section{LES STRUCTURES FUNÉRAIRES EN REMPLOI DE FOSSES DOMESTIQUES?}

Seuls quatre types de structure morphologiquement similaires aux fosses domestiques ont été utilisés à des fins funéraires: la fosse de grand diamètre peu profonde (GDPP), la fosse de petit diamètre peu profonde (PDPP), la fosse tronconique ou piriforme de type silo (SR) et la fosse de grand diamètre profonde, à paroi verticale (GDP). 


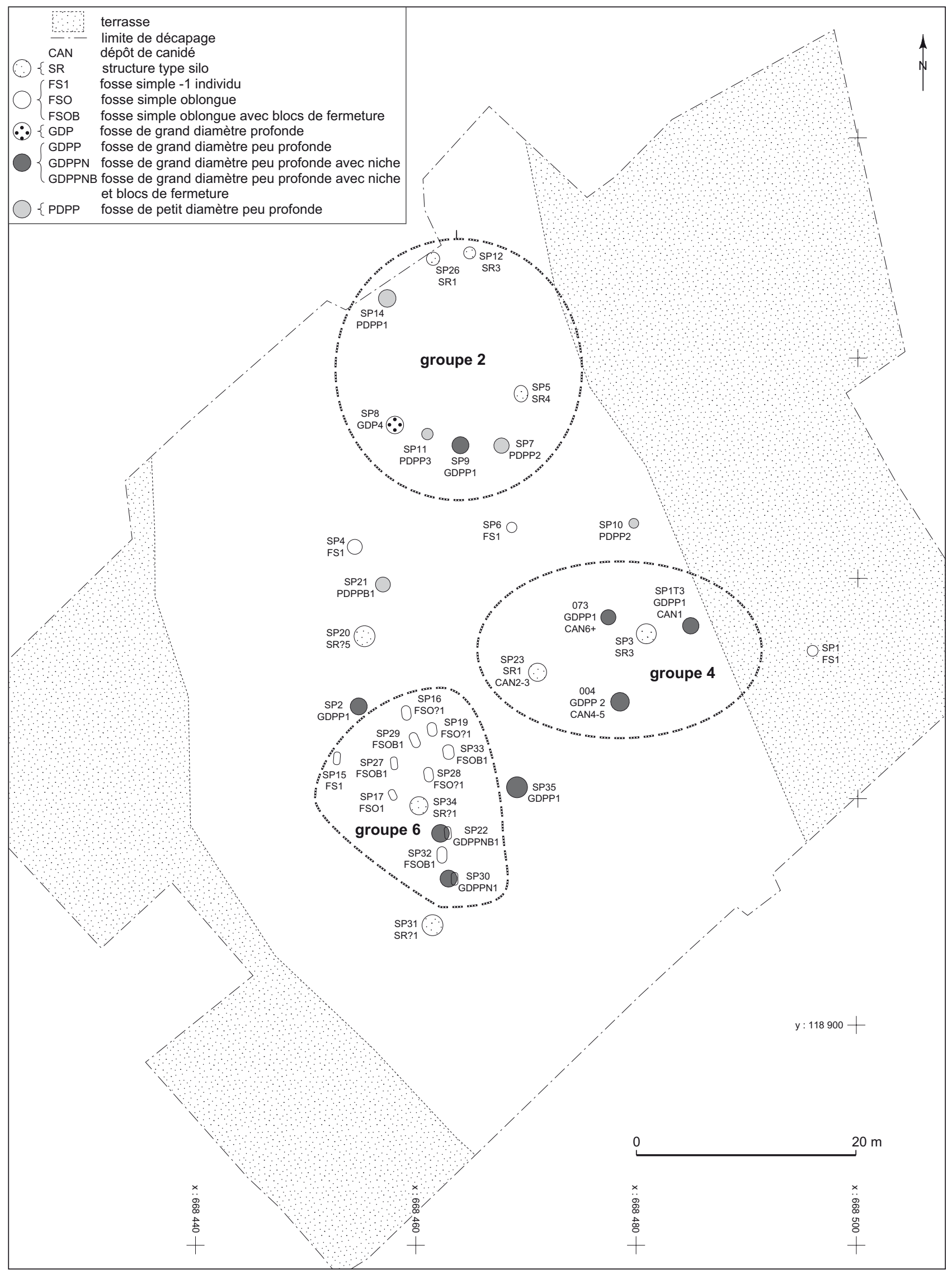

Fig. 3 - Les différents types de structures funéraires et sectorisation de l'espace (DAO: F. Audouit, C. Bioul, C. Labarussiat). 

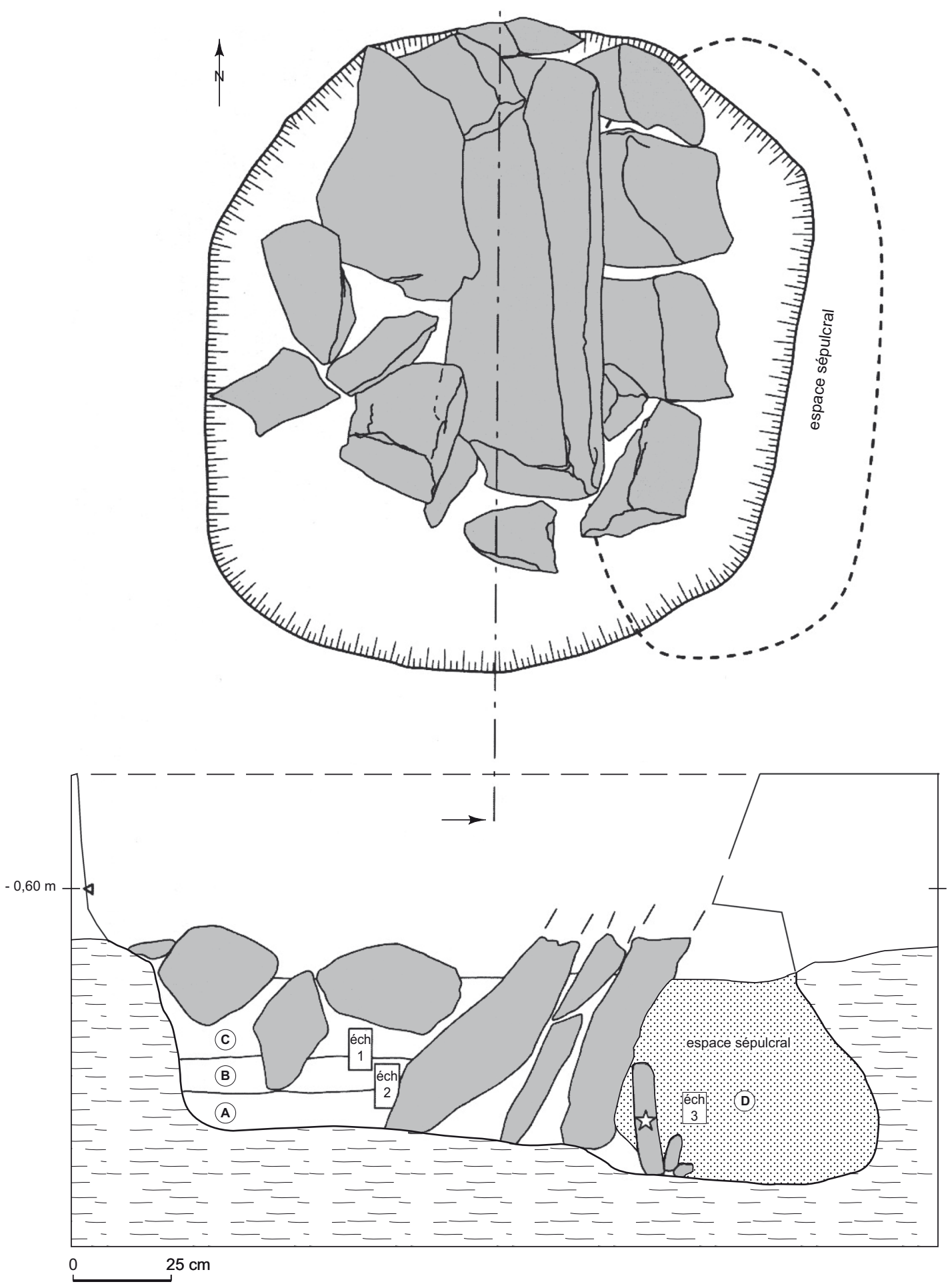

Fig. 4 - SP 22 - Fosse à cavité latérale: plan et coupe (DAO: S. Barbey). 


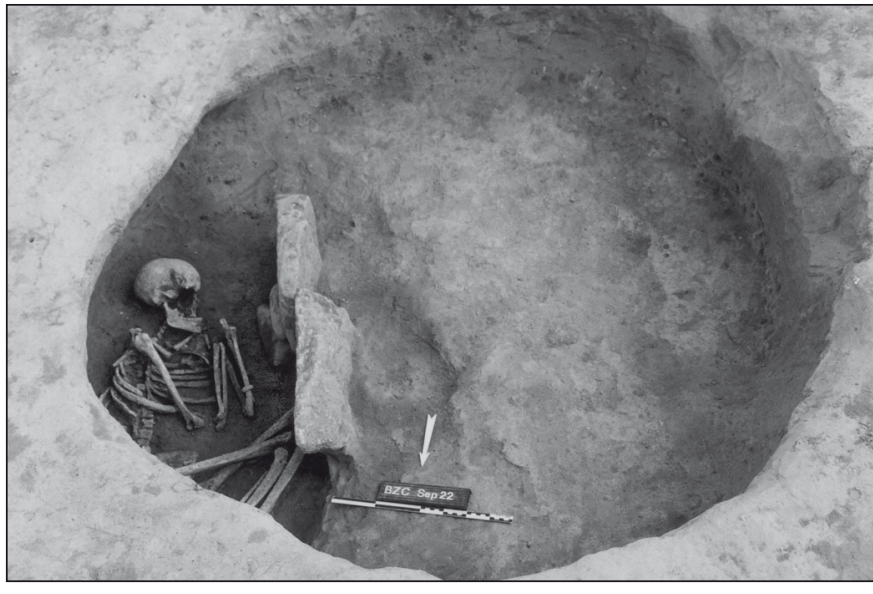

Fig. 5 - SP 22 - Inhumation dans la cavité sépulcrale.

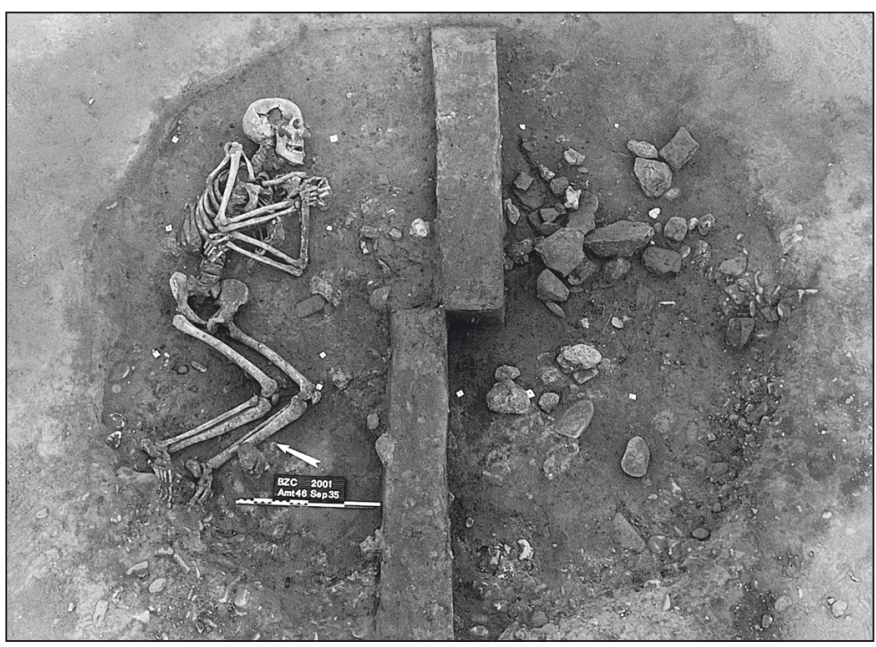

Fig. 6 - SP 35 - structure de type GDPP (fosse de grand diamètre peu profonde).

\section{La fosse de grand diamètre peu profonde (GDPP)}

Elle est représentée par 4 cas (SP 2, SP 9, SP 13, SP 35). Ces structures contiennent généralement un seul individu en position décentrée, seule une moitié de l'excavation semble préférentiellement utilisée, l'axe médian de séparation peut être nord-sud, ou est-ouest (fig. 6). Ces structures peuvent inclure un aménagement sommaire de blocs. Dans un seul cas, le dépôt d'un chien est associé à celui un individu humain (SP 13), selon une même partition de l'espace, les deux corps ayant été disposés tête-bêche à l'opposé l'un de l'autre. Ce type de fosse a également servi de réceptacle à deux inhumations de chiens disposées également tête-bêche à l'opposé l'une de l'autre (Amt 4).
En outre, dans cette catégorie de tombes, un dépôt simultané de deux enfants (SP 7) présentait des indices de combustion qui affectaient à la fois une loupe de sédiment qui recouvrait partiellement l'un d'eux et une partie des restes osseux. Cette pratique particulière, qui pourrait s'apparenter à une procédure de condamnation, est le seul cas de ce genre observé sur le site. Rappelons qu'à la même époque, en Roussillon, sur le site de Camp del Ginèbre à Caramany (Pyrénées-Orientales), la pratique de la crémation est attestée (Vignaud, 1998).

\section{La fosse sépulcrale de petit diamètre peu profonde (PDPP)}

Elle possède un diamètre compris entre $0,90 \mathrm{~m}$ et $1,30 \mathrm{~m}$, pour une profondeur allant de $0,24 \mathrm{~m}$ à $0,40 \mathrm{~m}$. Au nombre de 4, ces ensembles renferment quelquefois un seul individu (SP 14 et SP 21), mais aussi des dépôts multiples de 3 individus (SP 11) et successifs de 2 individus (SP 10); pour chacun des cas, la décomposition se serait effectuée en espace vide.

\section{La structure de type silo (SR)}

Elle est représentée par 6 ensembles (SP 3, SP 5, SP 12, SP 20, SP 23 et SP 26) et 3 morphologiquement plus discutables (SP 10, SP 31 et SP 34). Ces structures sont situées pour 3 cas dans le groupe 2 (SP 5, SP 12, SP 26), pour 2 cas dans le groupe 4 (SP 3 et SP 23), et un cas à la périphérie nord du groupe 6 (SP 20). Certaines ont livré des dépôts pluriels disposés selon un mode de fonctionnement complexe (SP 5, SP 12). Si quelques cas de dépôts multiples sont possibles (SP 20), la majorité des inhumations sont déposées successivement, intercalées entre des couches de comblement. Parmi ces dernières, certaines sont relativement anthropisées, mais on ne peut affirmer pour autant que ce sont assurément des rejets détritiques. Ces comblements, qui nous semblent de toute évidence volontaires, avaient probablement pour premier objet de séparer le dépôt du précédent. L'étude taphonomique des ensembles squelettiques et les observations microstratigraphiques suggèrent qu'un laps temps variable, mais parfois important, se serait écoulé entre les dépôts successifs, suffisamment long pour permettre une décomposition complète ou partielle du corps (fig. 7). Il s'agit bien là de dépôts successifs qui semblent s'organiser selon un schéma pré-établi. Le terme de «tombe à étages» a été employé pour caractériser ces dépôts (Chambon, Leclerc, 2007); 


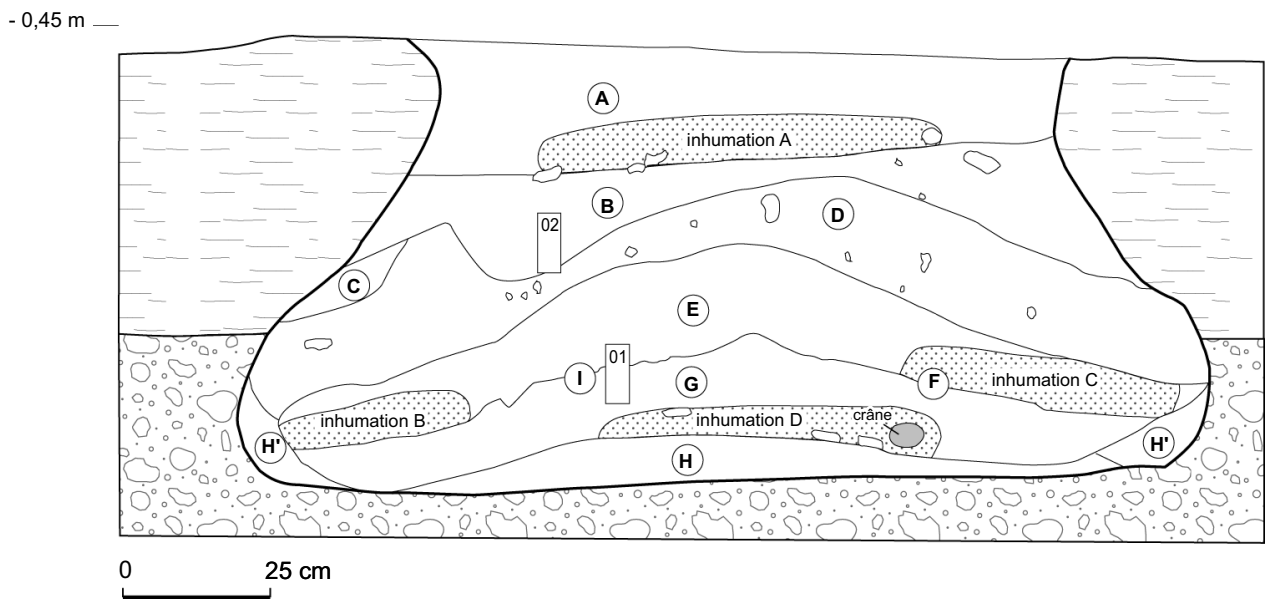

Fig. 7 - SP 5 - coupe stratigraphique (DAO: S. Barbey).

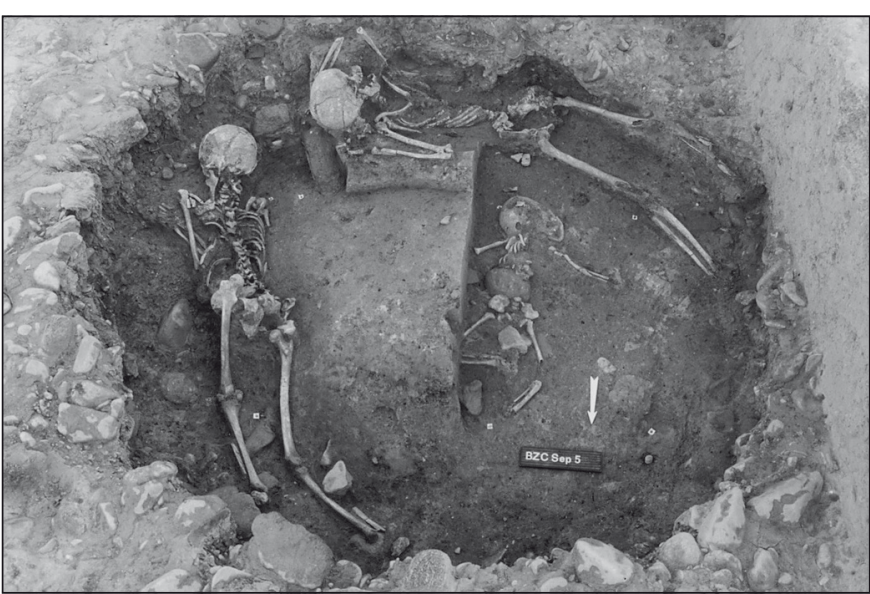

Fig. 8 - SP 5 - individus $B, C$ et $D$.

toutefois, celui de «tombe à dépôts étagés » nous semble dans ce cas plus adapté.

Ainsi, dans l'ensemble SP 5 (fig. 8), qui comporte 4 dépôts successifs, le premier inhumé est installé au centre de la fosse. Les suivants sont placés latéralement, à proximité de la paroi. Le décharnement s'est effectué dans la plupart des cas en espace vide, nécessitant la mise en place d'un dispositif d'obturation de la fosse, dont on peut imaginer qu'il devait être similaire à celui des silos utilisés pour la conservation des denrées alimentaires. Celui-ci a été réinstallé après chaque dépôt, car chacun des individus présente les caractéristiques de décomposition en espace vide. Enfin, dans ces structures, quelques défunts ont été déposés sur le dos et maintenus dans une position fortement contrainte («en grenouille») par un emballage relativement souple de type sac (en toile ou en peau?). Ce type de structure présente, dans son organisation, des similitudes avec la fosse 69 de Saint-Paul-Trois-Châteaux (Beeching, Crubézy, 1998, p. 152).

En Languedoc, en Provence et dans la vallée du Rhône, la sépulture individuelle ou plurielle en fosse de type silo est fréquente dans le Chasséen méridional où elle apparaît dès la phase ancienne (Ambert et al., 1988; Amiel, 1996; Vaquer, 1998; Vaquer, Duday 2003), localisée à proximité d'espaces domestiques (Loison et al., 2003 et 2004; Labriffe et al., 2007). La nature de ces fosses fait encore l'objet de débats: s'agit-il de silos réutilisés ou bien de fosses creusées en forme de silo, destinées à un usage sépulcral en «imitations de silos", terme utilisé par Alain Beeching à propos des sites plus récents de la moyenne vallée du Rhône (Beeching, 2007). La documentation détaillée recueillie sur le site du Crès et des observations similaires sur le site du Pirou à Valros (Loison, Gleize, à paraître) nous incite à retenir la première hypothèse.

Quoi qu'il en soit, ces structures ont un mode de fonctionnement qui s'inscrit dans la variabilité des pratiques funéraires des communautés chasséennes pour inhumer certains défunts. Et s'il s'agit, comme nous le pensons, d'un remploi de structures domestiques afin d'ensevelir certains défunts, ces deux fonctions ne s'opposeraient pas, mais se conjugueraient plutôt pour s'inscrire dans une symbolique particulière dont les liens sémantiques seraient la proximité de l'habitat et la fonction de conservation. La dimension «sacrée» de la fosse transformée en sépulcre est bien réelle: ce ne sont sans doute pas des sépultures de relégation et il est peu vraisemblable que ce remploi ne soit motivé que par une question d'économie de moyens. 
La fosse de grand diamètre profonde, à paroi verticale (GDP)

Elle n'est représentée que par un seul ensemble: SP 8. Les dépôts sont pluriels et parfois multiples. Comme pour les fosses funéraires de type silo, ils correspondent à des «dépôts étagés».

\section{GHRONOLOGIE ABSOLUE ET ORGANISATION SPATIALE DES STRUCTURES FUNÉRAIRES}

Les résultats de douze datations ${ }^{14} \mathrm{C}$ obtenues sur os sont d'une grande homogénéité, aucun ne montre de décalage anormal (fig. 9). Ces échantillons avaient été sélectionnés en tenant compte des caractères typologiques des tombes, de la représentativité du mobilier des structures et d'un premier schéma d'organisation du site.

Ces douze comptages radiocarbone présentent une marge d'incertitude relativement réduite: pour 2 à \pm 30 , pour 9 à \pm 35 , et pour 1 à \pm 60 . En années réelles (avec un intervalle de $95 \%$ de confiance), la présence chasséenne sur ce site s'inscrirait dans une fourchette probabiliste maximale de 4357 à 3996 av. J.-C. Cependant, un examen plus détaillé nous permet de distinguer trois séquences: une phase primitive comprise dans une fourchette de 4357 à 4167 av. J.-C., une phase moyenne entre 4331 et 4047 av. J.-C., et une phase terminale entre 4247 et 3996 av. J.-C. Les trois dates légèrement plus anciennes correspondent aux ensembles sépulcraux SP 2, SP 12, SP 22, auxquels pourrait s'ajouter un quatrième, SP 23, qui présente une marge d'incertitude plus grande.

Il en résulte que, dès la phase ancienne de l'occupation, les sépultures marqueraient déjà une grande diversité. Rappelons que la SP 12 est un ensemble sépulcral en fosse tronconique de type silo (type SR), comportant des inhumations plurielles, la SP 22 est une inhumation individuelle au sein d'une niche creusée dans la paroi d'une fosse de grand diamètre peu profonde de type GDPPNB (la chambre funéraire est semblable au type FSOB); c'est la tombe la plus monumentale du site. La SP 2 est une inhumation unique en fosse de grand diamètre peu profonde (type GDPP), des blocs de calcaire sont inclus dans le dispositif de condamnation; enfin, la SP 23 est un autre ensemble sépulcral complexe, en fosse tronconique de type silo (type SR), associant les dépôts de 2 chiens et 2 restes osseux isolés correspondant à 2 individus différents.

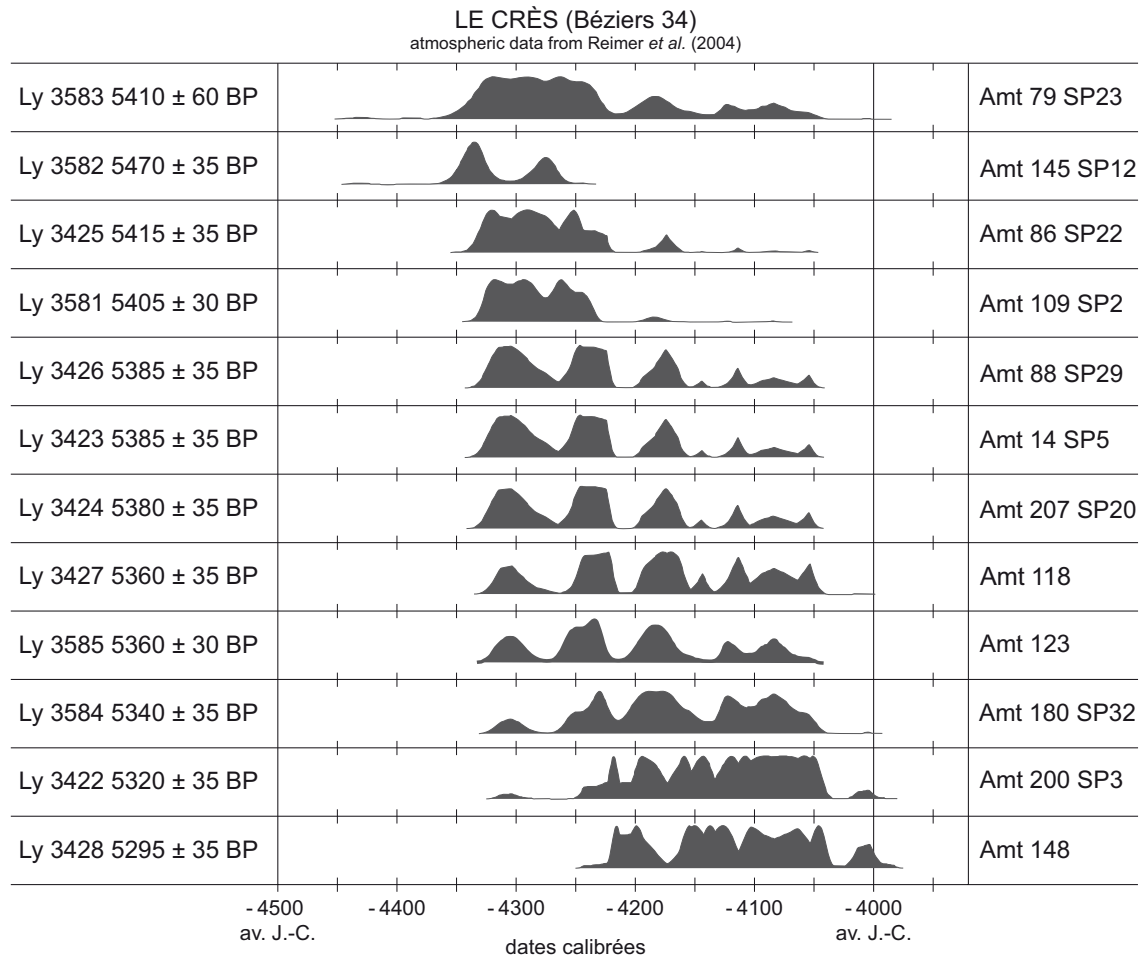

Fig. 9 : Datations ${ }^{14} C$. 
Ainsi, les types principaux de tombes, en fosse de type silo $(\mathrm{SR})$, en fosse de grand diamètre et en niche à accès latéral fermé par des dalles, coexisteraient dès le début de l'occupation. Il n'y aurait donc pas d'antériorité d'un type sur l'autre. De plus, les dépôts successifs en fosses de type silo avoisineraient les sépultures en fosse spécifiquement funéraires ne comportant qu'un seul individu, ainsi que les ensembles comportant des dépôts mixtes de canidés et de restes partiels d'humains.

Ces apports en chronologie absolue précisent la relative contemporanéité des inhumations, confirmant bien là l'étonnante diversité des pratiques funéraires relatives à la phase ancienne du Chasséen et, qui plus est ici, sur une même aire d'occupation.

En ce qui concerne l'organisation spatiale de ces ensembles funéraires, seul le groupe 6, constitué d'une concentration de fosses spécifiquement sépulcrales dont les plus nombreuses sont des sépultures individuelles en niche, peut être considéré comme un espace funéraire à part entière. Nous avions pu observer que ce secteur avait été préservé de tout autre type de creusement et que, parfaitement imbriqué dans la trame générale du site, il ne pouvait pas être postérieur aux autres groupes.

À la question de la coexistence sur un même site de pratiques funéraires apparemment aussi diversifiées, voire opposées, nous avions émis l'hypothèse que cet espace funéraire aurait pu être antérieur aux installations de nature domestique comme celles du groupe 2 et ses sépultures en fosse de type silo associées (SP 12 et SP 5) (Loison et al., 2004). Il n'en est rien, car la SP 22, qui semble avoir une position centrale dans ce groupe 6 , est même légèrement plus récente que la première. Cet espace funéraire ne peut donc pas être antérieur aux autres groupes. De plus, pour les 2 autres inhumations datées situées au sein de ce groupe 6 (SP 29 et SP 32), les dates obtenues seraient même un peu plus récentes que la SP 22, plaidant plutôt pour une pérennisation de cet espace spécifique.

Dans le groupe 4, qui a notamment la particularité de concentrer des inhumations de canidés, 2 ensembles funéraires ont fait l'objet de datations: la SP 23 et la SP 3. La première est proche de la phase ancienne, la seconde fait partie des plus récentes.

Bien que cette question nécessite encore quelques approfondissements, les résultats radiocarbone attesteraient que les groupes 2, 4 et 6 sont relativement contemporains, données qui vont dans le sens d'une confirmation de la permanence de l'occupation au Chasséen ancien et, en son sein, de la spécificité des certaines aires.

\section{LES PARAMĖTRES BIOLOGIQUES DE LA POPULATION INHUMÉE}

\section{PRÉAMBULE}

Dans les structures funéraires chasséennes, 49 sujets ont été individualisés. Quelques os isolés ont aussi été découverts dans ces contextes et il convient de discuter préalablement de leur statut dans le cadre de l'approche anthropologique.

Les ensembles SP 3 et SP 12 ont livré chacun une tête de fémur droit, dont la présence est anatomiquement incompatible avec les individus inhumés au sein de ces structures. La fosse SP 23 contenait également, en plus de 2 dépôts de chiens anatomiquement complets, 2 vestiges humains, une voûte crânienne immature et une prémolaire parfaitement mature. Quatre individus sont donc représentés par des pièces osseuses isolées. La présence d'os isolés est un phénomène largement connu dans la période chasséenne, dans des contextes divers (Pariat, 2007; Vaquer et al., 2008). Sur le site Les Moulins à Saint-Paul-Trois-Châteaux (Drôme) notamment, la fosse 69 , en plus des dépôts de 3 individus, a livré une extrémité distale d'un humérus d'enfant et les fragments d'un crâne d'enfant (Beeching, Crubézy, 1998). En admettant que, dans les structures funéraires du Crès, leur présence soit volontaire, leur statut est de toute évidence différent de celui des individus en position primaire. Nous avons donc préféré ne pas les intégrer dans notre corpus.

Dans la tombe SP 8, un bloc cranio-facial d'enfant décédé entre 6 et 10 ans était associé à l'individu $8 \mathrm{~A}$. Il s'agit ici d'un dépôt secondaire accompagnant le défunt qui ne fait donc pas partie de l'ensemble des individus inhumés en position primaire. Il était donc plus prudent de ne pas l'inclure dans notre échantillon de réflexion. Ainsi, le corpus à partir duquel nous allons analyser le profil de mortalité et le sex-ratio se compose de 44 individus.

\section{COMPOSITION DE LA POPULATION PAR SEXE}

Les méthodes proposées pour déterminer le sexe des individus non adultes ne sont pas fiables. Par conséquent, il n'est pas recommandé d'évaluer ce paramètre sur les sujets immatures (Bruzek et al., 2005). L'os coxal est l'élément du squelette le plus approprié pour déterminer le sexe. En effet, sa morphologie répond à des exigences adaptatives et fonctionnelles, liées à la locomotion et, surtout, à la reproduction (gestation et parturition), communes à 
toute l'espèce humaine. L'utilisation d'autres éléments du squelette n'est pas aussi fiable car le dimorphisme sexuel pris en compte n'est pas fondé sur la fonction, mais sur le format de cet os, caractéristique extrêmement variable selon les populations. Le sexe des individus adultes a été déterminé par deux méthodes utilisant les os coxaux: la méthode de diagnose sexuelle probabiliste (DSP), utilisant des données métriques (Murail et al., 2005), et la méthode visuelle reposant sur l'observation simultanée de la morphologie de 5 caractères (Bruzek, 2002). Cette méthode a été utilisée lorsque le minimum de 4 mesures, requis pour l'application de la DSP, n'était pas atteint.

La détermination du sexe a pu être réalisée sur 14 individus de taille adulte. La diagnose sexuelle probabiliste a été privilégiée, puisqu'elle est moins subjective et plus fiable que la diagnose morphoscopique. Elle a permis de déterminer le sexe de 10 individus. Toutefois, l'état de conservation a induit l'application de la méthode visuelle qui a permis de déterminer le sexe de 4 individus supplémentaires. L'étude en laboratoire a permis de mettre en évidence la présence de 8 femmes et de 6 hommes dans notre corpus d'individus adultes. Le sexe des individus S12 et S22 a été estimé sur le terrain avec certitude alors que l'état des os coxaux après prélèvement excluait la diagnose. Nous intégrons donc cette détermination à notre corpus: il s'agit respectivement d'un homme et d'une femme. Le sex-ratio est de 9 femmes pour 7 hommes (tabl. I). Le taux de masculinité est égal à 43,7\%, il indique une légère prépondérance des femmes, mais ce résultat n'est pas statistiquement significatif $(\mathrm{p}=0,723)$.

\section{COMPOSITION DE LA POPULATION PAR ÂGE}

La première étape consiste à estimer l'âge au décès de chaque individu.

\section{ESTIMATION DE L'ÂGE AU DÉCÈS DES INDIVIDUS IMMATURES}

Selon le stade de croissance, différentes méthodes ont été utilisées. L'utilisation de la croissance osseuse pour estimer l'âge au décès des enfants est fortement déconseillée. Toutefois, lorsque le décès est précoce, les facteurs génétiques et environnementaux n’ont pas eu le temps d'influencer le développement biologique d'un individu (Scheuer, Black, 2000). Par conséquent, la variabilité est faible pour les enfants de moins d'un an. L'estimation se fait en deux temps: la première étape évalue la stature à partir
Tabl. I - Estimation de l'âge au décès et détermination du sexe des individus.

\begin{tabular}{|c|c|c|c|}
\hline $\begin{array}{l}\text { Ensemble } \\
\text { sépulcral }\end{array}$ & $\mathbf{N}^{\circ}$ individu & Sexe & Âge estimé \\
\hline SP1 & 1 & femme & $>30$ ans \\
\hline SP2 & 2 & femme & $20-29$ ans \\
\hline \multirow{2}{*}{ SP3 } & $3 A$ & indéterminé & adulte \\
\hline & $3 B$ & indéterminé & 7 mois lunaires \\
\hline \multirow{4}{*}{ SP5 } & $5 \mathrm{~A}$ & indéterminé & adulte \\
\hline & $5 B$ & femme & $20-39$ ans \\
\hline & $5 \mathrm{C}$ & indéterminé & adulte \\
\hline & $5 D$ & indéterminé & $1,5-2,9$ ans \\
\hline SP6 & 6 & homme & adulte \\
\hline \multirow{2}{*}{ SP7 } & $7 \mathrm{~A}$ & indéterminé & $5,19-9,3$ ans \\
\hline & $7 \mathrm{~B}$ & indéterminé & $6,19-11,3$ ans \\
\hline \multirow{3}{*}{ SP8 } & $8 \mathrm{~A}$ & femme & adulte \\
\hline & $8 \mathrm{C}$ & indéterminé & $4,25-7,10$ ans \\
\hline & $8 \mathrm{D}$ & indéterminé & $4,25-7,10$ ans \\
\hline SP9 & 9 & femme & adulte \\
\hline \multirow{2}{*}{ SP10 } & $10 \mathrm{~A}$ & indéterminé & $17-18$ ans \\
\hline & $10 \mathrm{~B}$ & femme & $>50$ ans \\
\hline \multirow{3}{*}{ SP11 } & $11 \mathrm{~A}$ & indéterminé & $3,4-6$ ans \\
\hline & 11B & indéterminé & adulte \\
\hline & $11 \mathrm{C}$ & indéterminé & $3,4-6$ ans \\
\hline \multirow{2}{*}{ SP12 } & $12 \mathrm{~A}$ & homme & adulte \\
\hline & 12B & indéterminé & $5,13-8,57$ ans \\
\hline SP13 & 13 & indéterminé & $>60$ ans \\
\hline SP14 & 14 & indéterminé & adulte \\
\hline SP15 & 15 & indéterminé & adulte \\
\hline SP16 & 16 & homme & adulte \\
\hline SP19 & 19 & indéterminé & $1,5-2,9$ ans \\
\hline \multirow{5}{*}{ SP20 } & $20 \mathrm{~A}$ & indéterminé & $4,56-7,75$ ans \\
\hline & $20 B$ & indéterminé & $3,4-6$ ans \\
\hline & $20 C$ & indéterminé & $3,4-6$ ans \\
\hline & $20 \mathrm{D}$ & indéterminé & $4,56-7,75$ ans \\
\hline & $20 \mathrm{E}$ & indéterminé & $3,4-6$ ans \\
\hline SP21 & 21 & indéterminé & $8,35-13,6$ ans \\
\hline SP22 & 22 & femme & adulte \\
\hline SP26 & 26 & femme & adulte \\
\hline SP27 & 27 & indéterminé & $4,25-7,1$ ans \\
\hline SP28 & 28 & indéterminé & adulte \\
\hline SP29 & 29 & homme & adulte \\
\hline SP30 & 30 & femme & $>40$ ans \\
\hline SP31 & 31 & homme & $18-19$ ans \\
\hline SP32 & 32 & indéterminé & $>60$ ans \\
\hline SP33 & 33 & indéterminé & $6,19-11,3$ ans \\
\hline SP34 & 34 & homme & $>40$ ans \\
\hline SP35 & 35 & homme & $>40$ ans \\
\hline
\end{tabular}

de la longueur diaphysaire, pour laquelle sont appliquées les régressions de Sellier (1993). La seconde étape consiste à estimer l'âge à partir de la stature. Il est recommandé d'appliquer les régressions logarithmiques d'Olivier et Pineau (1958). 
Pour les individus de plus de un an, l'âge a été estimé par plusieurs méthodes. L'âge dentaire étant un indicateur plus performant que l'âge osseux (Saunders, 2000), il a été privilégié. Pour l'estimation à partir des dents, la méthode mise au point par Moorrees et ses collègues a été appliquée (Moorrees, 1963a et b). Lorsque les dents étaient absentes ou la minéralisation des dents permanentes complète, l'âge a été estimé à partir de la fusion des points d'ossification secondaire. De façon à ce que nos résultats puissent être comparés à d'autres études, l'estimation est donnée selon les tables proposées par Birkner (1980), qui présentent un équivalent chronologique au stade de maturation. Les résultats sont donnés dans le tableau I.

\section{ESTIMATION DE L’ÂGE AU DÉCÈS DES ADULTES}

L'estimation de l'âge au décès des adultes est un exercice difficile. La variabilité de la sénescence du squelette humain entre individus d'une même population et entre populations, due à une interaction entre le patrimoine génétique et le milieu environnant, induit un manque de précision et de fiabilité des méthodes (Bruzek et al., 2005). Les méthodes élaborées par A. Schmitt, basées sur la surface sacro-pelvienne iliaque et la symphyse pubienne, ont été appliquées (Schmitt, 2005 et 2008a). Elles tiennent compte de la variabilité intra- et inter-populationnelle européenne et privilégie la fiabilité à la précision. Les résultats figurent dans le tableau I.

\section{PRINCIPE DE L'ANALYSE DE LA COMPOSITION PAR ÂGE}

Cette étude est fondée sur la mise en évidence d'éventuelles anomalies dans le profil de mortalité de l'échantillon disponible (Sellier, 1996). En d'autres termes, il s'agit de vérifier si la population archéologique est assimilable à une population naturelle (Masset, 1987). La courbe de mortalité de la population inhumée est comparée à un «schéma de mortalité archaïque» qui se caractérise par une très forte mortalité infantile et par une espérance de vie à 20 ans plus élevée qu'à la naissance. Il est acceptable que, pour les populations du passé, l'espérance de vie à la naissance était comprise entre 20 ans et 40 ans (Masset, 1987 ; Sellier, 1993 et 1996).

La construction de la table de mortalité s'effectue par le calcul des quotients de mortalité par classes d'âges. Avant de comparer la répartition de ces derniers, il est souhaitable de calculer la proportion adulte/immature à partir du quotient de mortalité des moins de 20 ans $\left({ }_{0} q_{20}\right)$ pour voir s'il est conforme à celui d'une population préjennerienne (c'est-à-dire avant le premier vaccin contre la variole mis au point par E. Jenner en 1796). Ensuite, l'étude de la répartition des quotients de mortalité par classe d'âge permettra de mettre en évidence d'éventuelles anomalies démographiques. Cette analyse est fondée sur l'âge estimé des sujets immatures. En effet, l'effectif des adultes pour lesquels ce paramètre est quantifiable est faible. De plus, les estimations donnent des intervalles chronologiques trop larges pour permettre une telle étude.

Les non-adultes sont séparés en une classe annuelle ( 0 an) et en quatre classes quinquennales (1-4 ans, 5-9 ans, 10-14 ans et 15-19 ans). Il est ainsi possible de comparer les quotients de mortalité des sujets immatures inhumés avec ceux correspondant à une population préjennerienne dont l'espérance de vie à la naissance est estimée à 30 ans (Sellier, 1996). Les tables-types de Ledermann (1969) ont été choisies car elles ont été élaborées à partir de très nombreuses séries de conditions socio-économiques différentes et elles permettent d'obtenir des résultats avec un intervalle de confiance.

La répartition par classes quinquennales pose un problème lorsque l'estimation de l'âge des individus immatures chevauche deux classes. Notre but étant de mettre en évidence des anomalies majeures dans la répartition des classes d'âge, il est important de minimiser les différences entre la mortalité de la population et celle de référence. Cette nécessité a pu être résolue grâce à la minimalisation des anomalies, autrement appelée « principe de conformité»(Sellier, 1996).

Après avoir construit la courbe de répartition des quotients de mortalité par classes d'âge, il est possible d'observer les différences avec le profil provenant des tablestypes de Ledermann (1969). La mise en évidence d'anomalies doit ensuite être discutée: sélection des inhumés en fonction de l'âge, biais dus aux méthodes de fouille, crise de mortalité, etc. (Sellier, 1996).

\section{Profil de MORTAlité de LA SÉRIE DU GRÈS}

La plupart des fourchettes d'estimation obtenues pour les individus immatures proposent un résultat à cheval sur deux classes d'âge. Nous avons donc procédé à la minimalisation des anomalies démographiques. La combinaison retenue présentait la meilleure adéquation avec celle d'une courbe théorique. Le rapport de l'effectif décédé entre 5 et 9 ans sur celui décédé entre 10 et 14 ans doit être proche de deux et le rapport de l'effectif des sujets décédés entre 5 et 
Tabl. II - Répartition de la population immature en classes d'âge, selon le principe de la minimalisation des anomalies démographiques.

\begin{tabular}{|c|c|c|}
\hline Classe & Distribution réelle & Répartition finale \\
\hline 0 & 1 & 1 \\
\hline $1-4$ & 2 & 10 \\
\hline $1-4 / 5-9$ & 10 & \\
\hline $5-9$ & 2 & 5 \\
\hline $5-9 / 10-14$ & 3 & 2 \\
\hline $10-14$ & 0 & 2 \\
\hline $15-19$ & 2 & 24 \\
\hline $20+$ & 24 & 2,50 \\
\hline D5-9/D10-14 & & 0,29 \\
\hline D5-14/D20+ & & \\
\hline
\end{tabular}

Tabl. III - Table de mortalité de la série du Crès issue des effectifs bruts.

\begin{tabular}{|c|c|c|c|c|}
\hline $\begin{array}{c}\text { Âge } \\
\text { (années révolues) }\end{array}$ & $\begin{array}{l}\text { Décès } \\
\text { (D) }\end{array}$ & $\begin{array}{l}\text { Survivants } \\
\text { (S) }\end{array}$ & $\begin{array}{l}\text { Quotient } \\
\text { (q) }\end{array}$ & q en \%o \\
\hline 0 & 1 & 44 & 0,02 & 22,73 \\
\hline $1-4$ & 10 & 43 & 0,23 & 232,56 \\
\hline $5-9$ & 5 & 33 & 0,15 & 151,52 \\
\hline $10-14$ & 2 & 28 & 0,07 & 71,43 \\
\hline $15-19$ & 2 & 26 & 0,08 & 76,92 \\
\hline $20+$ & 24 & 24 & 1,00 & 1000,00 \\
\hline total & 44 & & & \\
\hline${ }_{0} q_{15}$ & & & 0,41 & 409,09 \\
\hline${ }_{0} q_{20}$ & & & 0,45 & 454,55 \\
\hline
\end{tabular}

\section{Quotient de mortalité (\%)}

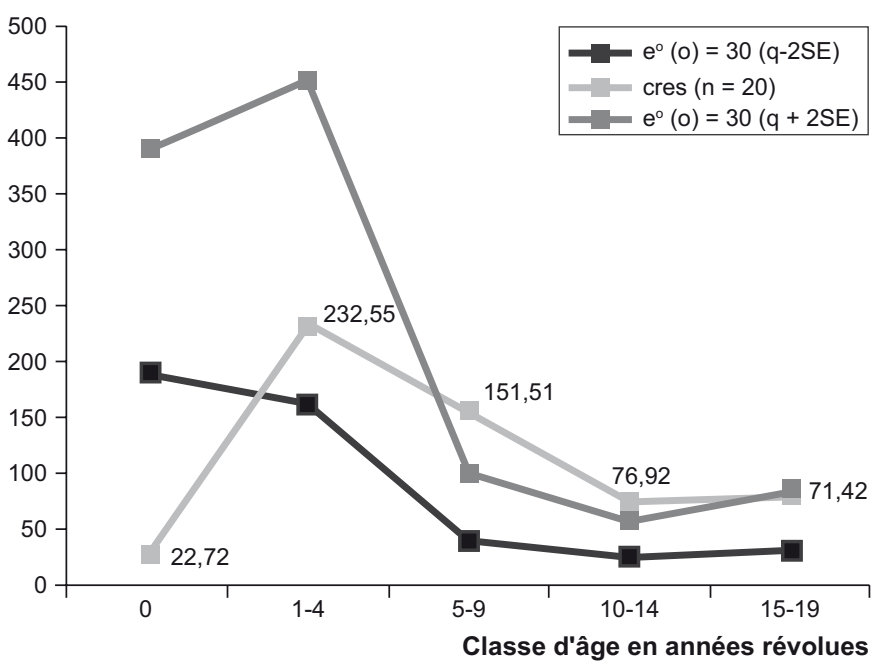

Fig. 10 - Comparaison des quotients de mortalité de la population du Crès avec les tables types de Ledermann (1969) pour une espérance de vie à la naissance de 30 ans.
14 ans et ceux morts après 20 ans doit être compris entre 0,1 et 0,3 (tabl. II). Le premier rapport, bien qu'étant le plus bas de toutes les combinaisons, fournit une valeur haute, mais raisonnable. Le nombre d'enfants décédés entre 5 et 14 ans est, par rapport au nombre d'adultes, conforme à la norme.

La distribution des individus immatures en fonction des cinq classes d'âge conventionnelles permet de construire la table de mortalité et de calculer les quotients de mortalité (tabl. III). Le quotient de mortalité avant 20 ans $\left({ }_{0} q_{20}\right)$ reflète la proportion des immatures par rapport à celle des adultes. Dans notre cas, il s'élève à $454 \%$ et est conforme au schéma de mortalité archaïque. En effet, celui des populations pré-industrielles est égal à $540 \%$, pour une espérance de vie à la naissance de 30 ans, avec des intervalles de confiance allant de $378 \%$ à $737 \%$. Le quotient de mortalité avant 15 ans, de $409 \%$ dans notre corpus, est également inclus dans les valeurs démographiquement acceptables comprises entre $360 \%$ et $714 \%$. Au premier abord, il n'y a pas de déficit des immatures par rapport aux adultes. Mais l'observation des quotients de mortalité pour chaque classe d'âge montre que le profil de mortalité n'est pas conforme à l'évolution du schéma théorique. Les quotients devraient être maximaux dans les deux premières classes, puis diminuer dans les deux suivantes et augmenter légèrement dans la cinquième, ce qui n'est pas le cas dans notre corpus. La représentation graphique (fig. 10) permet d'avoir une vision plus claire de ces anomalies. Le seul quotient de mortalité conforme est celui de la classe 1-4. La classe 0 est largement déficitaire. Les quotients des classes 5-9 et 10-14 sont trop élevés et celui de 15-19 est à la limite supérieure théorique.

\section{LES VARIATIONS ANATOMIQUES NON-MÉTRIQUES : RECHERCHE DE REGROUPEMENTS TOPOGRAPHIQUES}

Les variations anatomiques non-métriques sont des variations phénotypiques mineures, non pathologiques (Berry, Berry, 1967). Toutes ne sont pas héréditaires, les facteurs mésologiques ont parfois une responsabilité importante dans leur transmission ou dans leur apparition.

L'interprétation des fréquences des caractères non métriques est complexe car leur héritabilité est rarement connue. Actuellement, il semble qu'un déterminisme polyfactoriel est probable pour beaucoup d'entre eux (Saunders 1989; Crubézy et al., 1999). Certains sont liés à l'âge, au sexe ou bien à des facteurs extrinsèques (Hauser, De Stefano, 
1989; Crubézy, 1991b). Toutefois, l'héritabilité des variations non-métriques est considérée comme supérieure à l'héritabilité des caractères métriques (Scott, Turner, 1997; Crubézy et al., 1999). Il est également admis que les caractères discrets dentaires ont un fort déterminisme génétique (Scott, Turner, 1997; Larsen, 2002) puisqu'une fois la dent formée, sa morphologie est définitive.

Les variations anatomiques peuvent être utilisées pour l'étude des pratiques funéraires. En effet, une répartition spatiale non aléatoire des individus ayant en commun un ou plusieurs caractères anatomiques peut être interprétée en termes de regroupements issus d'une parenté biologique (Crubézy, Sellier, 1990).

Différents types de mise en évidence de regroupements topographiques existent. Toutefois, la projection graphique, si elle est subjective, n'est liée à aucune loi statistique de distribution de sujets (l'effectif de la série du Crès est trop faible pour procéder à des tests statistiques). De plus, elle présente l'avantage d'explorer aussi bien les relations à l'intérieur d'une même tombe qu'entre tombes adjacentes (Crubézy et al., 1999).

L'effectif des individus est plus élevé et plus pertinent sur le matériel dentaire. De plus, les caractères dentaires sont sous contrôle génétique. Pour la sélection, nous avons éliminé les caractères les plus rares (fréquence nulle ou 1 cas) et les plus fréquents (Schmitt, 2008b).

L'observation de la répartition spatiale des variations anatomiques est dictée par les données archéologiques. Pour le site du Crès, l'intérêt se focalise sur la proximité des tombes, mais également sur les structures ayant livré des inhumations plurielles. À cette échelle, une seule variation anatomique est circonscrite à deux tombes adjacentes (fig. 3). Il s'agit du tubercule de Carabelli sur la première molaire supérieure. Il a été observé sur les individus C et E de SP 20 et sur le sujet de l'inhumation individuelle SP 21. $\mathrm{Au}$ sein des tombes à dépôts étagés et/ou multiples, on observe des combinaisons de caractères partagées par des sujets inhumés dans la même entité funéraire. Dans SP 8, deux individus partagent trois caractères, le sillon coronaire de l'incisive latérale, le tubercule marginal de la deuxième prémolaire et les incisives centrales en pelle. Dans SP 20, les sujets $\mathrm{C}$ et $\mathrm{E}$ possèdent un tubercule de Carabelli sur la première molaire supérieure (comme l'enfant de SP 21 situé à proximité de SP 20) et des incisives centrales supérieures en pelle. Ces deux combinaisons ont été mises en évidence uniquement dans ces structures. Précisons que ces regroupements sont fondés sur des variations anatomiques nonmétriques dont le déterminisme génétique est réputé fort.

\section{CROISEMENTS DES DONNÉES ARCHÉOLOGIQUES ET ANTHROPOLOGIQUES}

L'un des objectifs de l'étude biologique a également été de fournir des paramètres supplémentaires pour contribuer à la compréhension du fonctionnement des ensembles funéraires.

En préambule, il convient de spécifier ce que nous entendons par enfants et adolescents. Précisons, d'ors et déjà, que nous parlons d'âge biologique. Toutefois, il n'y a pas de consensus sur ce sujet concernant les enfants (Halcrow, Tales, 2008). Ici, nous proposons d'utiliser ce terme pour les sujets décédés entre 1 et 14 ans. Au niveau biologique, l'adolescence est marquée par la maturité sexuelle. Par convention, il s'agit des individus décédés entre 15 et 19 ans (Bruzek et al., 2005).

\section{RÉPARTITION SPATIALE EN FONCTION DU SEXE ET DE L'ÂGE (FIG. 11)}

Dans l'approche générale sur l'organisation spatiale (cf. supra, Approches générales sur l'organisation spatiale), trois assemblages de structures funéraires ont été mis en évidence, correspondant aux groupes 2, 4 et 6 (fig. 3). Deux d'entre eux s'inscrivent au sein des groupes de fosses domestiques, considérés en tant qu'espaces domestiques spécifiques (groupes 2 et 4 ) et le groupe 6 est exclusivement constitué de structures funéraires.

Dans le groupe 2 (espace domestique), les individus, au nombre de 17, ont été inhumés au sein de 8 ensembles distincts (en fosses de nature domestique dont des fosses de type silo) : 5 sépultures plurielles et 3 sépultures individuelles. Les défunts correspondent à 8 enfants et 9 adultes (4 femmes, 1 homme et 4 indéterminés). Les enfants sont essentiellement présents dans les dépôts pluriels et associés dans la plupart des cas à des inhumations d'adultes.

Au sein du groupe 6, les individus inhumés sont au nombre de 11, et les sépultures sont essentiellement de type individuel. C'est dans ce groupe particulier que l'on rencontre des sépultures en cavité à fermeture latérale (en niche), obturée par des dalles et des blocs de calcaire. Celles-ci correspondent à 8 adultes ( 2 femmes, 3 hommes et 3 adultes indéterminés) et 3 enfants. Contrairement au groupe 2, même si les enfants sont présents, ils sont ici moins nombreux et, soulignons-le, tous en sépulture individuelle. Leurs modes d'inhumations sont identiques à ceux des adultes: en fosse simple ou en cavité latérale fermée par 


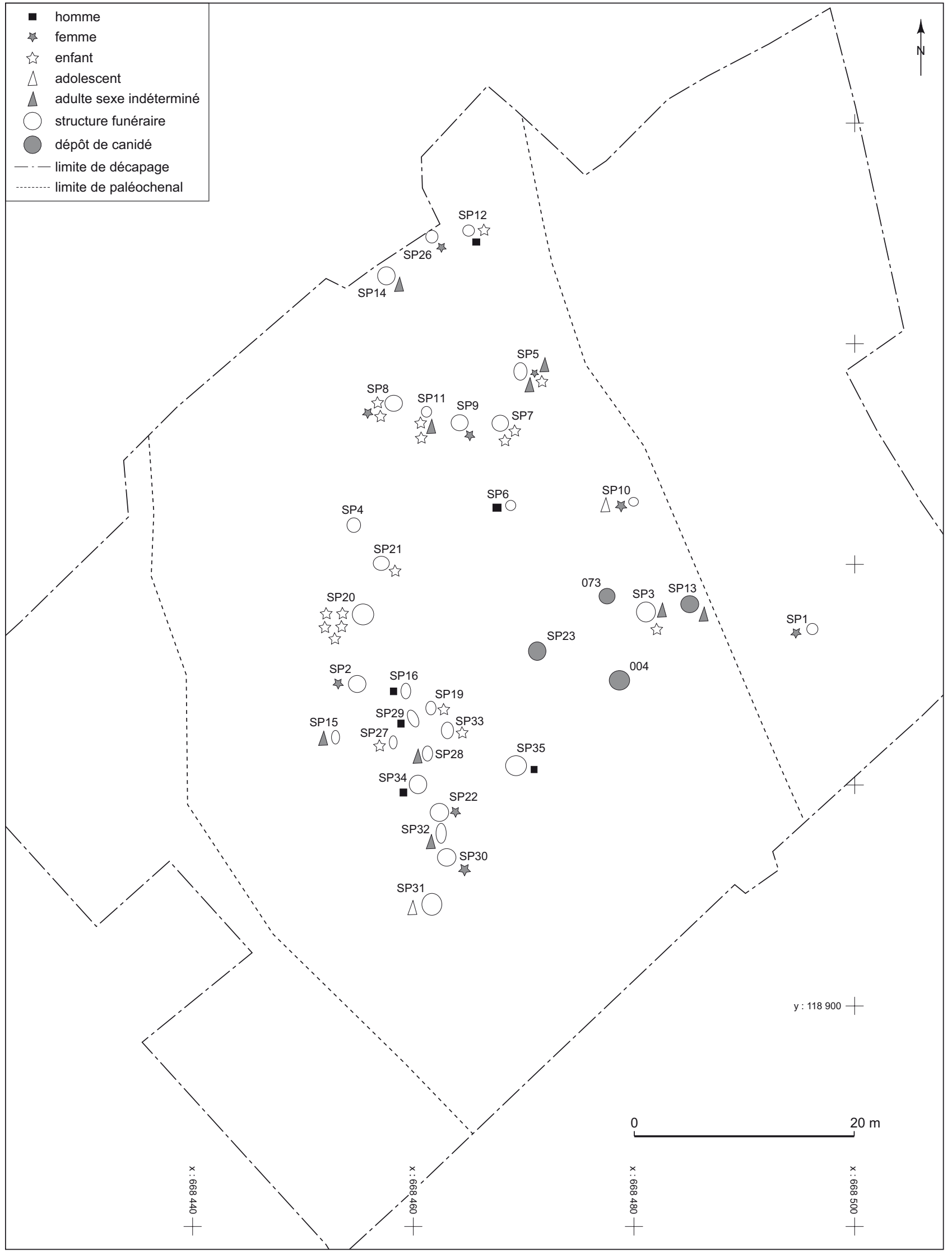

Fig. 11 - Projection sur plan des paramètres sexe et âge (enfant/adolescent/adulte) (DAO: A. Schmitt). 
des blocs. La présence exclusive de tombes dans cet espace réservé en marge des espaces domestiques nous autorise à le considérer comme un petit cimetière.

Dans le groupe 4, les individus inhumés sont au nombre de 3, disposés au sein de 2 ensembles distincts qui sont des sépultures plurielles. Les défunts correspondent à 2 adultes de sexe indéterminé et 1 foetus. Toutefois, la particularité de cet espace est la présence de chiens, au nombre de 6. Ces animaux ont non seulement été inhumés dans des fosses voisinant celles des humains, en dépôts simples et doubles mais, dans deux cas, ils ont été associés à des restes humains au sein d'une même fosse (SP 13 et SP 23). Dans cet espace, la présence exclusive de canidés, que ceux-ci soient associés ou non à des humains, confère à ce lieu un caractère spécifique, qui semble avoir fonctionné pendant toute la durée de l'occupation.

Quant aux ensembles SP 2, SP 20 et SP 21, qui se situent spatialement entre les groupes 2 et 6 , il n'est pas exclu qu'ils puissent être des «satellites » de l'un ou de l'autre. Les individus inhumés sont au nombre de 7 (6 enfants et 1 femme). Les enfants ont été déposés au sein de deux ensembles distincts: une sépulture individuelle et un dépôt pluriel de 5 enfants au sein d'une grande fosse de type silo. Sa particularité réside dans le fait qu'aucun adulte n'y est associé.

\section{LES ANOMALIES DÉMOGRAPHIQUES}

Comment expliquer les anomalies démographiques observées ? L'existence d'un biais méthodologique, lié à une erreur dans l'estimation de l'âge, est peu probable puisque la distribution des individus en classes ne pose pas de problèmes s'ils sont ventilés selon le principe de minimalisation des anomalies démographiques. Un seul individu décédé entre 0 et 1 an a été mis au jour (SP 3). Il était associé à un individu adulte. Le déficit de cette classe d'âge est relativement courant dans les populations néolithiques (Loison, 1998). Cette question ne peut être résolue par la seule explication d'une mauvaise conservation des sujets les plus jeunes. Nous optons plutôt pour une réelle absence. La sous-représentation des individus décédés entre 0 et 1 an est probablement le résultat du choix d'un autre lieu de sépulture ou d'un autre mode de gestion des corps pour cette catégorie d'âge. Notons, toutefois, que la présence d'un fotus dans la structure SP 3 montre qu'ils peuvent être inhumés dans des fosses de type silo et qu'ils ne sont donc pas complètement exclus de l'espace funéraire. En revanche, comment expliquer le sureffectif des enfants décédés entre 5 et 14 ans? Pour tenter de répondre à cette question, il est nécessaire de préciser leur situation dans l'organisation du site. Précisons que ces individus immatures ont été ventilés dans une seule classe d'âge. Cette distribution effectuée de manière statistique peut ne pas correspondre à proprement parler à une vraie distribution. Il faut, donc raisonner sur l'ensemble des tombes recevant des enfants. Au sein du groupe 2, 5 structures funéraires sur 8 ont accueilli des dépôts pluriels d'enfants dont une sépulture double (SP 7) et une triple (SP 11). Les inhumations SP 5, SP 8et SP 12 sont des "tombes à dépôts étagés". Ces dépôts pluriels sont constitués de 9 adultes et 8 enfants, dont un décédé en bas âge et 7 pouvant appartenir soit à la classe 5-9, soit à la classe 10-14. A contrario, dans le groupe 6, comparativement à l'effectif des adultes, les enfants, au nombre de 3 , sont sous-représentés. La sépulture SP 20, proche du groupe 6, de type fosse-silo, est un cas spécifique. Elle ne contient que des enfants, au nombre de 5 , dont 2 inhumations simultanées d'enfant des classes 5-9 ou 10-14.

Treize des dix-huit enfants ont été inhumés dans des structures dont la première vocation serait domestique, par opposition au groupe 6 , au sein de tombes à dépôts étagés ou simultanés. Au Crès, ces inhumations simultanées, qui concernent principalement la population des enfants, restent minoritaires. B. Boulestin propose que, si les individus sont inhumés ensemble, soit un décès est la cause des autres, soit les décès ont la même cause (Boulestin, 2008). Le premier cas de figure correspond à l'accompagnement (Testart, 2004), le second à des événements particuliers de type crise de mortalité, qu'elle qu'en soit l'échelle (Boulestin, 2008). Évoquer de tels phénomènes de crise au Néolithique est justifié, mais cerner leur origine s'avère problématique (Crubézy, 1991b). La mortalité excédentaire, observée sur le site du Crès, pourrait correspondre, par exemple, à une épidémie de faible amplitude. Notons, par ailleurs, que dans SP 7 et SP 11, structures renfermant des dépôts simultanés, on observe que les défunts présentent des différences de posture et de disposition dans l'espace qui pourraient être liées à une différence de statut social des individus les uns par rapport aux autres, sans toutefois être en mesure d'affirmer qu'il s'agit de morts d'accompagnement.

\section{LE MODE D'INHUMATION EST-IL LIÉ AU SEXE ET À L'ÂGE DES INDIVIDUS?}

Si la comparaison des traitements funéraires entre sexe et âge est limitée, dans la mesure où aucun test statistique ne peut être mené, nous pouvons, néanmoins dégager des tendances. 


\section{QUELQUES DONNÉES SUR L'ANALYSE TAPHONOMIQUE}

L'analyse taphonomique des restes osseux a permis de mettre en évidence la présence d'éléments de contention du corps tels que ceux exercés par un emballage ou des vêtements pour 18 individus ( $\mathrm{SP}$ 2, SP 3-ind.A, SP 5-ind.A, B et C, SP 10-ind.A et B, SP 11-ind.A, B et C, SP 14, SP 21, SP 22, SP 26, SP 30, SP 31, SP 33 et SP 34). Il semblerait qu'il y ait plusieurs types d'enveloppes ou de liens, différents de par leur nature (cuir, tissu...), leur fonction et leur résistance à la putréfaction.

Parfois, plusieurs types d'enveloppes semblent se combiner autour d'un même défunt. Certaines inhumations laissent entrevoir la possibilité d'au moins deux enveloppes de nature différente recouvrant l'ensemble du corps: peut-être un vêtement et un emballage funéraire proprement dit. D'autres fois, la présence d'un second élément semble plus localisée. Dans ce cas, les pieds ont été rassemblés au niveau du bassin, liés l'un à l'autre, avant d'être enveloppé à l'aide d'une matière souple et suffisamment épaisse pour pouvoir assurer une bonne tenue (peau, nappe...). Cette hyper-contraction (position «en grenouille»), en relation directe avec un empaquetage du corps, a été observée sur les ensembles SP 14, SP 5-ind.A, et SP 10-ind.A. Sur ce site, elle ne concerne que les sujets déposés sur le dos.

Le dernier inhumé de la fosse EDF 6 du Gournier à Montélimar (Drôme), individu en position hyper-contractée, et peut-être celui en position similaire dans la fosse HS5 de ce même site, interprété comme étant positionné dans un coffre aux dimensions restreintes (Beeching, Crubézy, 1998, p. 154-155), pourraient correspondre à ce même type de pratique, bien qu'ils soient ici déposés sur le côté.

Par ailleurs, l'emballage des corps concerne 12 adultes ( 6 femmes, 1 homme et 5 indéterminés) pour 2 adolescents ou jeunes adultes et 4 enfants. Il semblerait que l'emballage des corps soit préférentiellement, mais non exclusivement, destiné aux femmes plutôt qu'aux hommes, mais un biais important est amené par les adultes de sexe indéterminé. Pour les enfants, hormis le foetus de SP 3, cette pratique ne semble pas réservée à une catégorie d'âge particulière.

\section{COMPARAISON ENTRE SEXES}

Au sein du groupe 6 (ensemble funéraire spécifique), aucune différence de traitement funéraire des adultes n'a été observée selon le sexe, selon la position et la situation du corps dans l'espace de la fosse. En revanche, les deux fosses à cavité latérale (SP 22, fig. 4 et 5 et SP 30) ont été destinées aux femmes. L'analyse du groupe 2 est limitée, puisqu'on ne peut raisonner que sur un homme et quatre femmes. Toutefois, le traitement funéraire du sujet masculin de SP 12 est similaire à celui de la femme (ind.B) de SP 5 (fig. 8, à gauche). Les deux structures sont des fosses de type silo, les inhumations sont successives, la position des corps se distingue de celle des individus inhumés au centre des fosses, qui s'apparente à celle du groupe 6 .

\section{COMPARAISON ENFANTS/ADOLESCENTS/ADULTES}

La disposition des corps et leur orientation est variable (Fabre et al., 2004). Les positions les plus répandues sont le dépôt sur le dos ou sur le côté gauche. La moins courante est le dépôt sur le côté droit. Cette dernière semble réservée aux adultes ou apparentés. De même, le côté gauche n'est que rarement appliqué aux enfants (SP 20-ind.C et SP 33).

L'orientation des dépôts présente également une grande variabilité et les différences observées semblent plus tenir du type de structure funéraire que tout autre critère directement lié à l'identité du défunt. Notons que, lorsqu'une même structure renferme plusieurs individus en dépôts successifs ou simultanés, l'orientation des corps n'est jamais la même et, dans certains cas, elle semble s'organiser en fonction des dépôts précédents (SP 5).

Les deux adolescents sont inhumés dans des structures de remploi domestique. Dans SP 31, le sujet est seul, il est légèrement décentré, sur le dos, les jambes fléchies sur le côté gauche, les membres supérieurs ramenés devant le menton. La sépulture se situe au sud et à la périphérie du groupe 6. Le traitement s'apparente à celui de plusieurs adultes de ce groupe. Le second adolescent (SP 10-ind.A) a été inhumé dans la même fosse qu'une femme de plus de 50 ans, mais les deux dépôts sont successifs. Sa position s'apparente à celle de l'adulte inhumé dans SP 14. On peut donc envisager que le traitement des adolescents ne se distingue pas de celui des adultes si on situe l'analyse à l'échelle des groupes 2 et 6.

Au sein du groupe 6 , il y a trois sépultures individuelles d'enfants (SP 19, SP 27, SP 33). La mauvaise conservation de SP 19 et SP 27 ne permet pas de discuter de leur position. Les sujets de SP 27 et SP 33 ont été inhumés dans une fosse à cavité latérale à l'instar des deux femmes de SP 22 et SP 30. Dans le groupe 2, les enfants sont aussi nombreux que les adultes. Ils se situent toujours dans des structures à dépôts étagés et/ou simultanés et, au sein de ces fosses, il y a toujours au moins un adulte (sauf dans SP 7). Dans SP 7 
et SP 8, ils sont déposés par deux, simultanément. Dans SP 8, les deux enfants sont dans la même tranche d'âge, malheureusement la mauvaise conservation ne permet pas de connaître leur position initiale. Si le corps de l'individu C recouvrait partiellement l'individu $\mathrm{D}$, la simultanéité de ces dépôts n'est pas assurée. La structure SP 11 comportait trois corps en position superposée dont deux enfants de la même tranche l'âge dans une position similaire, sur le ventre. Dans SP 7, a contrario, la position est complètement différente, le premier inhumé, légèrement plus âgé que le second, a été déposé sur le dos, légèrement à l'est de la fosse. Le second défunt a été placé sur les membres inférieurs du précédent contre la paroi sud. Dans ce cas, on peut émettre l'hypothèse que la différence de position et de situation dans l'espace de la fosse peut être induite par l'âge et/ou un statut social différent. Dans les structures à dépôts étagés, l'enfant de SP 12 a été déposé au centre de la structure sur le dos, les membres inférieurs légèrement fléchis sur le côté gauche. Le sujet SP 5-ind.D est le premier inhumé d'une fosse de type silo renfermant quatre individus. Il repose sur le dos, les membres supérieurs écartés du corps en extension et les cuisses et les jambes légèrement fléchies, au milieu de la fosse.

Dans SP 20, située entre les groupes 2 et 6, cinq enfants, décédés entre trois et huit ans, ont été mis au jour sur, au moins, deux niveaux distincts. La contemporanéité du dépôt des sujets $\mathrm{A}$ et $\mathrm{B}$, au premier niveau d'inhumation, est clairement établie, les corps se superposent partiellement au niveau des membres inférieurs. L'enfant de SP 21 adjacente à SP 20 repose sur le ventre, dans la moitié ouest de la fosse.

Pour les enfants, au sein des structures dont la première vocation était domestique, la diversité du traitement des corps et le type des structures d'accueil ainsi que l'organisation en leur sein, ne se distinguent pas de la variabilité observée pour les adultes inhumés dans le groupe 2 .

Plusieurs combinaisons de variations anatomiques nonmétriques ont été observées sur plusieurs individus au sein d'une même structure (SP 8, SP 20) On peut donc envisager que dans ces deux ensembles sépulcraux, des défunts ont, pour le moins, des liens de parenté biologique, ce qui ne signifie pas que ce rapprochement est valable pour les autres structures à dépôts pluriels. De plus, la sélection des défunts ne reposait probablement pas sur cet unique paramètre. Deux individus de SP 20 et le sujet SP 21 possèdent une variation anatomique dentaire présente uniquement dans ces deux structures. Ces enfants partageaient également une parenté biologique. Or, ces deux structures sont adja- centes, le rapprochement des défunts peut donc être lié à ce paramètre. L'information pertinente, ici, est la différence entre le traitement funéraire de défunts apparentés.

Au sein des groupes 2 et 6 , la diversité ne semble pas être liée à l'âge et au sexe. Toutefois, les tombes à niches latérales complexes sont destinées aux femmes et on ne peut exclure que la différence de traitement entre les deux enfants de SP 7 soit, en partie, liée à l'âge. Dans le groupe 2, la variabilité des modes d'inhumation traduit probablement une organisation sociale complexe. Les deux ensembles distincts, 2 et 6 , sont, comme nous l'avons vu, marqués par des modes funéraires très différents et contemporains. Ceux-ci ne semblent pas se distinguer sur des critères biologiques: dans les deux groupes, nous trouvons une présence aussi bien des adultes des deux sexes et des enfants, (même si les enfants sont plus nombreux dans le groupe 2), mais plutôt sur des critères sociaux dont l'origine est, à ce stade, hypothétique.

\section{LE MOBILIER D'ACCOMPAGNEMENT}

Il se compose principalement de récipients en céramique, d'outils en os (lissoir, poinçon), de parures en coquillage, de valves de moules utilisées en tant qu'outils, d'armatures de flèches, et de matériaux de mouture. Sur l'ensemble des inhumations de ce site, 15 comportaient un mobilier d'accompagnement, constitué généralement de une ou deux pièces. Cependant, dans quelques cas, certains objets trouvés dans l'environnement des défunts ne présentaient pas toutes les garanties d'une association directe, ceux-ci pouvaient tout aussi bien avoir été introduits lors des comblements, notamment dans le cas des inhumations en fosses de type silo; ils n'ont donc pas été pris en compte dans cet inventaire.

La situation de ce mobilier ne montre pas de discrimination systématique, mais certaines associations présentent cependant un caractère assez spécifique et quelques particularités. Ce mobilier accompagne 10 adultes (4 femmes, 2 hommes et 4 adultes indéterminés), 1 adolescent et 4 enfants, correspondant aux différentes classes d'âge démographiques. Il se rencontre dans 6 fosses de type silo (SR), 5 tombes en niche (FSOB), 3 fosses de petit diamètre peu profonde (PDPP) et 2 de grand diamètre peu profonde (GDPP).

Nous ne présentons pas ici un inventaire détaillé par tombes, mais seulement une évocation générale par nature. Précisons cependant que seuls trois ensembles comportent des dépôts multiples. Il s'agit des sépultures: SP 2, femme 
en fosse GDPP (1 vase ovoïde, 1 poinçon et 1 spatule en os, 1 meule plate et 1 petit bloc mortier), SP 33, un enfant en niche ( 2 armatures triangulaires en silex, 1 perle discoïde en callaïs, 1 spatule en os et 1 un petit bloc mortier) et SP 3, adulte indéterminé en fosse de type silo (4 outils en os et 1 pendeloque sur canine de porcin).

La céramique n'est présente (probablement en tant que contenant) que dans deux ensembles funéraires, l'un en fosse simple (SP 15) ou en GDPP (SP 2). Toutefois, la tombe SP 11, à inhumations multiples, contenait les fragments d'un vase brisé disposés au-dessus des restes osseux.

Les 5 armatures triangulaires exhumées (Léa, 2004), proviennent majoritairement de tombes du groupe 6 (SP 28, 29, 33), la SP 33 en comportait 2. Elles sont associées à un homme, un adulte indéterminé et un enfant. Une seule a été découverte dans le groupe 2 (SP 12) sur le niveau de dépôt de l'individu B; nous ne sommes cependant pas certain que cette présence soit intentionnelle.

Les outils en matière dure animale sont plus nombreux : sur les 45 outils ou pièces prélevés, 22 sont issus des contextes funéraires (Gaillard, 2004). La majorité provient d'os de ruminants domestiques, $45 \%$ sont des outils perforants et $40 \%$ mousses ou frottants. Ces outils sont présents dans les tombes en fosse de type silo (SP 3, 12, 26 et 31), dans celles de type GDPP (SP 2 et 9 ) et dans celles de type PDPP (SP 10 et 14), associés à 4 enfants et 6 adultes ( 4 femmes et 2 indéterminés). Bien que les deux indéterminés biaisent quelque peu cette information, il semble donc que ces outils n'accompagneraient pas (ou peu) les hommes. Signalons une pièce sur côte de grand ruminant mise au jour dans la SP 26 qui, bien que de dimensions plus réduites, peut être comparée à l'«épée» de Najac (Hérault) ou à des pièces similaires de Villeneuve-Tolosane (Vaquer et al., 2008).

En outre, 7 outils étaient associés à des dépôts de chiens (Amt 4, 73 et 79), l'Amt 4 en présentait à elle seule 4 exemplaires. Cette constatation semble bien accréditer le fait que, dans un certain nombre de cas, il s'agit bien de sépultures.

Les parures sont, sur ce site, peu représentées (Forest, 2004), elles sont associées à seulement 4 ensembles funéraires (SP 13, 19, 29, 33). Pour la SP 19, la plus remarquable, il s'agit de quatre pendentifs, situés au niveau du thorax d'un enfant et constitués de 3 valves de cardium perforées dans la convexité du sommet et d'un pendentif en forme de «gâchette» taillé dans l'épaisseur d'une coquille d'huître. Il peut s'agir des éléments d'un collier, mais cet assemblage singulier peut aussi avoir eu pour objet d'émettre un tintement (de type hochet). Dans la SP 29, un pendentif arqué en forme de goutte, confectionné également sur une coquille d'huître et perforé transversalement, a été trouvé sous l'arcade zygomatique gauche du défunt. Une perle discoïde en callaïs faisait partie du mobilier de la SP 33.

Il est important de préciser que les inhumations, comportant des pendeloques, figurent dans les structures en niche appartenant au groupe 6, elles sont associées à deux enfants et un homme. La seule exception est la présence d'une valve de cardium perforée déposée dans la SP 13 (fosse de type GDPP).

Le matériel de mouture et de broyage, témoin d'activité domestique, est présent dans les tombes, entier ou fragmenté. Il se compose de meules plates de taille variable ou de molettes en grès et de dallettes ou galets plats en roche dure ayant servi de mortier. Ce matériel a été retrouvé en association directe (SP 2, 5, 30, 32, 30) et indirecte, inclus dans l'amas de pierres scellant l'inhumation (SP 9) ou dans une couche sous-jacente au dépôt (SP 14). Les meules plates sont parfois associées à l'organisation interne du dépôt; ainsi dans la fosse de type silo (SP 5) à dépôts pluriels, une grande meule brisée a été déposée au niveau du premier inhumé (un enfant). Elle repose de chant au fond de la fosse, appuyée par une partie latérale, contre la paroi de la fosse. Une couche de sédiment a scellé le premier dépôt, laissant visible le sommet de cette meule. Deux autres défunts (une femme et un adulte de sexe indéterminé) ont été installés successivement de part et d'autre de cette meule en position opposée (au niveau des crânes) : il semble très probable qu'elle ait servi de démarcation. Dans la structure en niche (SP 22), une meule plate complète, disposée de chant et calée par un broyon en quartzite, forme avec une autre dalle le dispositif de fermeture latéral de la tombe. D'autres objets appartenant à cette catégorie ont été découverts en association directe avec des défunts, il s'agit d'une dallette ou galet plat ayant servi de mortier. Ceux-ci sont présents dans les tombes individuelles SP 30 et 32, de type en niche, appartenant au groupe 6 et dans la SP 2, tombe située à la périphérie nord du groupe 6 (de type GDPP). Cet outillage est systématiquement retrouvé sous l'un des avant-bras. Des dépôts, semble-t-il de même nature, ont été mis au jour sur le site du Gournier à Montélimar (Drôme), également dans des sépultures individuelles ES4 et ES31 (Beeching, Crubézy, 1998, p. 157).

A. Beeching voit dans cette omniprésence du matériel de mouture dans le rituel funéraire non pas une preuve d'agriculture céréalière, mais une compensation à une dégradation agricole liée à la montée en puissance d'un 
pastoralisme itinérant (Beeching, 2003). Au Crès, la forte présence de fosses de type silo et la présence récurrente de céréales carbonisées au sein des strates sédimentaires ne nous autorisent pas à aller dans ce sens.

D’un point de vue plus général, ces objets de mouture et de broyage sont associés à 5 femmes et 2 adultes indéterminés, ainsi que, dans la SP 5, à un enfant mais, dans ce cas, ce dépôt semble avoir servi de préparation pour les inhumations suivantes où l'on trouve une femme ainsi qu'un adulte indéterminé. Par ailleurs, ces matériaux de mouture comme les outils sur matière dure animale ne seraient pas présents dans les tombes des adultes masculins, mais pourraient être principalement associés aux adultes féminins.

Enfin, d'autres dépôts peuvent aussi être apparentés à du mobilier funéraire, il s'agit de la présence singulière dans les tombes de certaines pièces osseuses d'animaux, principalement de bœuf (scapula, pattes, crâne ou fragment de crâne...). On les rencontre exclusivement dans les fosses domestiques de remploi, principalement dans les fosses de type silo, associés aussi bien à des sépultures humaines qu'à des chiens. Leur évidente proximité avec certains dépôts funéraires nous autorise à considérer ces pièces, non pas comme de simples rejets, mais bien, en raison de leur nature, de leur situation particulières et de leur répétitivité, à les reconnaître comme de réels dépôts chargés de symbolique. Cette question a été discutée notamment à propos du site du Gournier à Montélimar (Drôme). A. Beeching insiste sur le statut particulier et le rôle que les bovinés ont joué dans les représentations symboliques de la Préhistoire récente méditerranéenne (Beeching, 2003).

Ce rapide examen du mobilier funéraire nous montre que celui-ci est relativement modeste par rapport à celui retrouvé sur les sites des phases plus récentes et qu'il inclut principalement des objets du quotidien. Cependant, les quelques tendances dégagées nous apportent des éléments significatifs sur l'organisation sociale et symbolique de cette communauté représentative du complexe chasséen. Les armatures triangulaires ainsi que les éléments de parure, tout modestes qu'ils soient, sont principalement associés aux tombes du groupe 6, espace exclusivement sépulcral. L'outillage en matière dure animale semble destiné aux femmes, aux enfants et à certains chiens, plutôt qu'aux hommes; de plus, il n'est présent que dans les fosses funéraires d'origine domestique. Enfin, l'association de matériel de mouture aux tombes d'adultes de sexe féminin pose la question d'une spécialisation par sexe de certaines activités domestiques.

\section{BILAN}

Plusieurs auteurs ont avant nous fait le constat d'une étonnante diversité des structures et des pratiques funéraires chasséennes (Boujot et al., 1991) qui semblait interdire tout essai d'établissement d'un cadre culturel ou évolutionniste simple (Gallay, 1991). La coexistence de pratiques et de types de tombes aussi diverses dans le Chasséen méridional avait été également soulignée par J. Vaquer; elle semblait révélatrice, selon l'auteur, d'une complexité de l'idéologie funéraire chasséenne (Vaquer, 1998).

L'approche que nous présentons du site du Crès a eu pour but de faire état des premiers résultats disponibles issus du nécessaire, et un peu tardif, croisement des données archéologiques avec celles de l'étude anthropologique revisitée et des datations radiocarbone. Ces résultats, encore préliminaires, permettront cependant d'alimenter les réflexions sur ce phénomène funéraire particulièrement complexe.

Pour ce premier niveau d'interprétation, et afin de ne pas rester dans des généralités, il a été nécessaire d'apporter un certain nombre de précisions qui concernaient: la nature de l'occupation, la constitution des vestiges et leur organisation à partir d'une définition typologique des structures, leurs relations spatiales et chronologiques, la situation des ensembles sépulcraux, le recrutement funéraire, la représentativité des modes de dépôts. Dans un second temps, nous avons tenté de préciser ce que l'échantillon du Crès pouvait apporter au décryptage du système funéraire du Chasséen méridional.

Il est possible dès lors de dégager quelques tendances:

- Par la nature des vestiges structuraux et mobiliers, l'occupation du Crès est, pour partie, celle d'un site d'habitat, où des regroupements particuliers de fosses ont pu être mis en évidence. Ils sont considérés comme des aires domestiques dans lesquelles des défunts ont été inhumés, mais un espace spécifiquement funéraire coexiste à proximité.

- Les datations radiocarbone précisent la relative contemporanéité des inhumations mises au jour sur ce site, confirmant l'étonnante diversité des pratiques funéraires relatives à la phase ancienne du Chasséen et, qui plus est ici, sur une même aire d'occupation.

- L'étude du recrutement de la population inhumée montre que $54 \%$ de la population est adulte (24 adultes pour 20 individus immatures), proportion d'adultes acceptable d'un point de vue démographique. Par contre, parmi 
la population des immatures, la classe d'âge 0 est anormalement déficitaire, alors que les individus décédés entre 5 et 14 ans sont, eux, surreprésentés. Étant donné que plusieurs enfants sont inhumés simultanément, cette mortalité excédentaire pourrait correspondre à une épidémie de faible amplitude.

- Les structures funéraires se répartissent en trois concentrations distinctes, plus ou moins denses, qui s'inscrivent dans le schéma d'organisation général. Deux d'entre elles sont situées au sein des groupes de fosses domestiques, considérés en tant qu'espaces domestiques spécifiques (groupes 2 et 4) et une, dans le groupe 6, constituée exclusivement de structures funéraires.

Il semble que l'âge (enfant/adolescent/adulte) et le sexe des sujets n'étaient pas des critères discriminants pour le choix de la tombe et du mode d'inhumation. L'étude sur la répartition spatiale des défunts en fonction du sexe et de l'âge conclut qu'aucune distribution spatiale particulière en fonction de ces deux paramètres n'a été mise en évidence. Notons, toutefois, que les enfants sont minoritaires dans le groupe 6 .

La particularité du groupe 4 est la présence de sépultures de chiens, au nombre de 6. Ces animaux ont, non seulement, été inhumés dans des fosses voisinant celles des humains, en dépôts simples et doubles mais, dans deux cas, ils ont été associés à des dépôts humains au sein d'une même fosse (SP 13 et SP 23). La présence exclusive de chiens, associés ou non à des humains, témoigne d'une dévolution spécifique de ce lieu. Si l'on peut être assuré du caractère sépulcral de ces dépôts - des canidés ont bénéficié des mêmes attentions que certains humains (emballages, espaces réservés) - il conviendra de préciser leur statut: sépultures animales ou morts d'accompagnements?

Les dépôts de chiens comme au Crès ne sont pas des cas isolés dans le Chasséen méridional, et plus particulièrement dans sa phase ancienne; ils ont notamment été mis en évidence sur le site Les Plots à Berriac dans l'Aude (Vaquer, 1998) et plus récemment sur le site du Pirou à Valros (Hérault), distant de $20 \mathrm{~km}$ du Crès. Toutefois, le fait nouveau est que nous pouvons affirmer que certains de ces animaux du Crès ont bénéficié d’un espace réservé et d'un traitement funéraire semblable à celui pratiqué sur les défunts humains.

- Le mobilier accompagnant les défunts est principalement composé d'objets usuels: récipients en céramique, outils en os (lissoir, poinçon), valves de moules utilisées en tant qu'outils, armatures de flèches, matériaux de mouture, ainsi que quelques rares pendentifs sur coquillage et un en callaïs.

Ces dépôts, souvent simples ou doubles, ne présentent pas de distinction particulière liée au sexe ou à l'âge, mais sont sensiblement plus fréquents parmi les adultes que chez les enfants. Confrontée aux différents types de structures d'accueil et de pratiques funéraires, leur distribution ne semble pas refléter de marque de statut particulier. Seules trois inhumations présentent des dépôts multiples (SP 2, SP 3, SP 33), mais elles ne diffèrent cependant pas typologiquement des autres ensembles.

On note en revanche, d'un groupe à l'autre, quelques éléments de distinction, comme la présence des armatures triangulaires ainsi que des éléments de parure, principalement associés aux tombes du groupe 6, espace exclusivement sépulcral. L'outillage en matière dure animale, quant à lui, semble destiné aux femmes, aux enfants et à certains chiens, plutôt qu'aux hommes; de plus, il n'est présent que dans les fosses funéraires d'origine domestique.

À partir des observations faites au Crès à Béziers, les éléments caractéristiques des pratiques funéraires du Chasséen ancien languedocien peuvent se définir de manière plus précise.

- Une catégorie de défunts est inhumée dans des fosses domestiques, présentant un lien de proximité avec des aires d'habitats, dont certaines (notamment de type silo) ont pu faire l'objet d'un remploi. Il est donc possible d'affirmer que certains défunts sans discrimination d'âge et de sexe ont bien été inhumés en contexte domestique. Il peut s'agir d'inhumations individuelles ou plurielles. Dans le second cas, elles sont le plus souvent successives (dépôts étagés séparés par des strates de sédiments), quelquefois multiples, et comportent généralement deux individus; un seul cas, la SP 11, en comporte trois. Pour les structures de type silo, l'espace funéraire permet, tout comme un caveau, le décharnement des corps en espace confiné et les dépôts successifs et ordonnancés des défunts. Une des hypothèses serait que certaines de ces structures pourraient être liées à une gestion de type familiale, ce qui n'exclut pas d'autres critères de sélection.

- Les observations taphonomiques sur les restes osseux et la mise en évidence de certaines manipulations d'ossements témoignent que la plupart de ces structures ont été obturées par un dispositif qui a permis la décomposition de chacun des corps en espace confiné.

- Pour les dépôts simultanés de SP 7 et SP 11 (la mauvaise conservation des pièces osseuses limite la lecture 
de SP 8 et SP 20), la position et la situation des défunts dans la fosse indiquent une différence de traitement, peut-être liée à une différence de statut social des individus inhumés ensemble.

- Dans le cas de dépôts successifs, la configuration la plus courante est celle qui rassemble un individu en position centrale et un ou plusieurs individus dans une position que l'on peut considérer comme subordonnée, mais qui en réalité résulterait d'un schéma pré-établi. Certains auteurs voient dans ces individus en position subordonnée des «morts d'accompagnement» (Testart, 2004). Ce concept est novateur pour l'étude des pratiques funéraires du Néolithique moyen, mais il faut cependant se méfier d'un emploi trop systématique en vue d'expliquer les sépultures multiples (Boulestin, 2008) ; cela peut par ailleurs tendre à masquer d'autres réalités. Ainsi au Crès, si l'on tient compte de la succession des dépôts, il ne peut s'agir que «d'accompagnants différés ».

- Les sépultures en fosses en milieu d'habitat, spécificité du Chasséen méridional, semblent se généraliser dès la deuxième moitié du $\mathrm{V}^{\mathrm{e}}$ millénaire avant notre ère, jusqu'à devenir un phénomène transculturel, dont on observe la présence notamment en contexte Michelsberg voire NMB ou même dans le Valais, mais là probablement en contexte chasséen (Mariéthoz, 2007). Certains auteurs vont jusqu'à parler de province funéraire (Boulestin, 2008).

- Il existe aussi un autre type d'inhumation: la sépulture individuelle en fosse simple oblongue et en niche dont, pour ces dernières, l'accès latéral est fermé par des dalles de calcaire. Ces tombes sont regroupées sous la forme d'un petit cimetière dissocié des aires d'habitat, elles témoignent d'analogies typologiques avec le complexe culturel catalan des sepulcros de fosa. Le mobilier qui leur est associé présente des différences avec celui des sépultures de l'espace domestique, il est principalement composé d'armatures triangulaires et d'éléments de parure.

Si ce site répond de manière satisfaisante à la question des tombes en milieu d'habitat, il a mis en évidence, au sein d'une surface relativement restreinte $\left(5000 \mathrm{~m}^{2}\right)$, cette dichotomie déjà remarquée entre les tombes en milieu d'habitat, que l'on peut sans conteste attribuer aux traditions chasséennes, et les tombes architecturées en nécropole. Si les tombes en habitat ont récemment été confirmées par la découverte du Pirou à Valros (Hérault), il n'est pas interdit d'imaginer qu'il existe d'autres petites nécropoles dissociées ou proches des habitats, à l'image des tombes de la station du Moulin de Sauret à Castelnau-LeLez dans l'Hérault (Audibert, 1956), du monumental site de Caramany (Pyrénées-Orientales), de Pontcharaud (Puy-deDôme), voire de l'ensemble du Gournier (Drôme, tombes du monument E-F), bien que ce dernier appartienne à une époque plus récente du Chasséen.

Cette coexistence de modes funéraires et d'espaces aussi variés, en un même lieu et à une même période, soulève naturellement des interrogations sur la structuration sociale de cette population, dont l'étude anthropologique a montré une relative homogénéité biologique (Schmitt, 2008). Ceci nous conduit à penser que de telles différences dans le traitement des morts peuvent être l'expression d'une société stratifiée, dans laquelle les membres ont des statuts distincts et hiérarchisés, statuts pouvant tout aussi bien être de nature socio-économique que symbolique. Ces questions seront abordées dans nos prochains travaux portant sur la question des sépultures en fosses comme marqueurs culturels et sociaux au Néolithique moyen, recherche qui sera menée principalement à partir des sites du Crès à Béziers, du Pirou à Valros (Hérault) et de Pontcharaud à Clermont-Ferrand (Puy-de-Dôme).

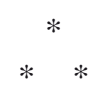

Confrontées aux premières études archéologiques, les données nouvelles présentées ici ont permis d'apporter des précisions sur l'organisation du site et sur ce que cette découverte peut apporter à la connaissance du Chasséen méridional, notamment en ce qui concerne les relations entre les ensembles sépulcraux et les structures domestiques. Il est indéniable que les découvertes réalisées sur ce site offrent des éléments de première importance pour le renouvellement des connaissances de ce sujet complexe et encore trop peu documenté.

Nous avons pu mettre en évidence un certain nombre d'énoncés nouveaux de cette grammaire funéraire propre au Chasséen méridional, régie comme nous l'avons vu par un ensemble de pratiques et probablement de codes complexes, dont beaucoup nous sont encore hermétiques. Et bien que nous pensons en avoir dégagé quelques tendances, l'ordonnance syntaxique reste encore à appréhender. 


\title{
BIBLIOGRAPHIE
}

\author{
AbRÉVIATIONS \\ APRAIF Association pour la recherche archéologique en Île-de-France. \\ BSPF Bulletin de la Société préhistorique française. \\ BSR Bilan scientifique régional. \\ CTHS Comité des travaux historiques et scientifiques. \\ DAF Documents d'archéologie française. \\ MSH Maison des sciences de l'homme. \\ RFO Rapport final d'opération. \\ SPF Société préhistorique française. \\ SRA Service régional de l'archéologie.
}

\section{Ambert P., Genna A., TAFFanel $O$.}

1988: «Contribution à l'étude du Chasséen du Minervois: le Chasséen en Languedoc», in Hommage à Jean Arnal, Actes des Journées d'Études de Montpellier, oct. 1985, Préhistoire UPV $n^{\circ} 1$, Publication de la recherche, Université Paul Valery, p. 25-36.

AMIEL C.

1996: «Quarante, Encombres», BSR du Languedoc-Roussillon, p. 134.

AUdibert J.

1956: La Station du Moulin de Sauret (Castelnau-le-Lez, Hérault), BSPF, 53, p. $402-404$

\section{BEECHING A.}

1991: «Sépultures, territoires et société dans

le Chasséen méridional: l'exemple du bassin rhodanien", in BEECHING A. (DIR.), Identité du Chasséen, Nemours, APRAIF (coll. Mémoires du Musée de préhistoire d'Île-de-France, 4), p. 327-341.

2003: "Organisation spatiale et symbolique du rituel funéraire chasséen en moyenne vallée du Rhône: première approche", in Chambon P., LeClerC J. (DIR.), Les Pratiques funéraires néolithiques avant 3500 av. J.-C. en France et dans les régions limitrophes, Paris, SPF (coll. Mémoires de la SPF, XXXIII), p. 231-239.

2007: «L'affaire «Chamblandes»: ramifications chrono-culturelles et géographiques du dossier, in Chambon P., Moinat P. (DIR.), Les Cistes de Chamblandes et la place des coffres dans les pratiques funéraires du Néolithique moyen occidental, Lausanne, Cahiers d'archéologie romande (coll. Cahiers d'archéologie romande, 110 et Mémoires de la SPF, XLIII), p. 69-74.

BeEching A., CRUbÉZY E.

1998: «Les sépultures chasséennes de la vallée du Rhône», in GUILAINE J. (DIR.), Sépultures d'Occident et genèses des mégalithismes (9000-3500 avant notre ère), Séminaire du Collège de France, Paris, Errance, p. $147-164$

BERRY A., BERRY R. J.

1967: «Epigenetic variation in the human cranium", Journal of Anatomy, 101, p. $361-379$.

BIRKNER R.

1980: L'Image radiologique typique du squelette, Paris, Maloine, traduit de l'allemand par C. Bléry-Lüft, revu par M. Bléry (édition originale: Das typische Röntgenbild des Skeletts, Munich, Urban \& Schwarzenberg, 1977).

Boujot C., CRubéZy E., BEeching A.

1991: "L'identité du Chasséen à travers les structures et les pratiques funéraires» in BEECHING A. (DIR.), Identité du Chasséen, Nemours, APRAIF (coll. Mémoires du Musée de préhistoire d'Île-de-France, 4), p. $413-420$

\section{BOULESTIN B.}

2008: «Pourquoi mourir ensemble? À propos des tombes multiples dans le Néolithique français ", $B S P F, 105$, p. 103-130.

BOSCH J., FAURa J.-M.

2003: "Pratiques funéraires néolithiques dans la région des bouches de l'Ebre», in Chambon P., LeClerC J. (DIR.), Les Pratiques funéraires néolithiques avant 3500 av. J.-C. en France et dans les régions limitrophes, Paris, SPF (coll. Mémoires de la SPF, XXXIII), p. 153-158.

\section{BRUZEK J.}

2002: «A method for visual determination of sex, using the human hip bone»,
American Journal of Physical Anthropology, 117 , p. $157-168$

BruzeK J., Schmitt A., Murail P.

2005. «Identification biologique individuelle en paléoanthropologie: détermination du sexe et de l'âge au décès à partir du squelette", in DUTOUR O, HUBLIN J.-J., VANDERMEERSCH B. (DIR.), Objets et méthodes en Paléoanthropologie, Paris, CTHS, p. 217-245.

\section{Chambon P., Leclerc J.}

2007: «Les tombes multiples dans le Néolithique français: aléa statistique ou pratique institutionnalisée?", BSPF, 104, p. 289-306.

\section{COURTIN J.}

1974: Le Néolithique de la Provence, Paris, Klincksieck (Mémoires de la SPF, 11), 359 p.

\section{CRUBÉZY E.}

1991a: Caractères discrets et évolution: exemple d'une population nubienne, Thèse de doctorat, Univ. Bordeaux-1, 684 p.

1991b: «Les pratiques funéraires dans le Chasséen de la moyenne vallée du Rhône», in BEECHING A. (DIR.), Identité du Chasséen, Nemours, APRAIF (coll. Mémoires du Musée de préhistoire d'Îlede-France, 4), p. 393-398.

Crubézy E., Sellier P.

1990: "Caractères discrets et organisation des ensembles sépulcraux", Bulletins et Mémoires de la Société d'anthropologie de Paris, 2, p. 171-178.

Grubézy E., Telmon N., Sevin A., Picard J., Rougé D., Larrouy G., BRAGa J., Ludes B., MURAIL P.

1999: Microévolution d'une population histori- 
que, Paris, Société d'anthropologie de Paris (coll. Bulletins et Mémoires de la Société d'anthropologie de Paris, nouvelle série, 11), $213 \mathrm{p}$.

\section{D'ANNA A}

1993: «L'habitat de plein air en Provence: recherches récentes ", in Le Néolithique au quotidien, Actes du XVI colloque interrégional sur le Néolithique, Paris, 5-6 nov. 1989, Paris, éd. de la MSH (coll. DAF, 39), p. 72-84.

\section{DUDAY H.}

1987: "Contribution des observations ostéologiques à la chronologie interne des sépultures collectives", in DUDAY H., MASSET C. (DIR.), Anthropologie physique et archéologie: méthodes d'études des sépultures, Actes du colloque de Toulouse, 4-6 nov. 1982, Paris, éd. du CNRS, p. 51-59.

\section{FABRE V., LOISON G., VILLEMEUR I.}

2004: «Les ensembles sépulcraux néolithiques", in LOISON G., VABRE V., VIllemeur I., Rocade Nord de Béziers (Hérault): Le Crès, habitats préhistoriques en bordure de l'Orb, RFO, SRA LanguedocRoussillon, p. 181-305 (74 fig., 3 tabl.).

\section{FOREST V.}

2004: «Étude archéozoologique», in LOISON G., VABRE V., VIllemeUR I., Rocade Nord de Béziers (Hérault): Le Crès, habitats préhistoriques en bordure de l'Orb, RFO, SRA Languedoc-Roussillon, p. $390-408$.

\section{GAILlaRD A.}

2004: «Inventaire et étude préliminaire du mobilier en matière dure animale», in LOISON G., VABRE V., VIllemeuR I., Rocade Nord de Béziers (Hérault): Le Crès, habitats préhistoriques en bordure de l'Orb, RFO, SRA Languedoc-Roussillon, p. $277-287$

\section{Gallay A}

1991: «L'homme néolithique et la mort», Pour la Science, 164, p. 78-87.

2006: Les Sociétés mégalithiques: pouvoir des hommes, mémoire des morts, Lausanne, Presses polytechniques et universitaires romandes (coll. Le savoir suisse, 37), $139 \mathrm{p}$.

\section{HaUser G., De STEFano G.F.}

1989: Epigenetic Variants of the Human Skull, Stuttgart, Schweizerbart, $301 \mathrm{p}$.

HALCROW S. E., TAYLES N.

2008: «The bioarchaeological investigation of childhood and social age: problems and prospects», Journal of Archaeological Method and Theory, 15, p. 190-215.

\section{JEDIKIAN G.}

2004: «Étude préliminaire du mobilier céramique", in LOISON G., VABRE V., VILlemeur I., Rocade Nord de Béziers (Hérault): Le Crès, habitats préhistoriques en bordure de l'Orb, RFO, SRA LanguedocRoussillon, p. 311-353.

LAbriffe (DE) P. A., LOISON G., LÉA V., HASLER A.

2007: «De la fosse au mégalithe, de l'individuel au collectif: les constructions funéraires au Néolithique moyen en Languedoc oriental et en Provence», in Chambon P., MoInAT P. (DIR.), Les Cistes de Chamblandes et la place des coffres dans les pratiques funéraires du Néolithique moyen occidental, Lausanne, Cahiers d'archéologie romande (coll. Cahiers d'archéologie romande, 110 et Mémoires de la SPF, XLIII), p. 27-39

\section{LARSEN C. S.}

2002: «Bioarchaeology: The Lives and Lifestyles of Past People", Journal of Archaeological Research, 10, p. 119-166.

LÉA V.

2004: «Étude technologique de l'industrie lithique taillée», in LOISON G., VABRE V., VILlemeuR I., Rocade Nord de Béziers (Hérault): Le Crès, habitats préhistoriques en bordure de l'Orb, RFO, SRA LanguedocRoussillon, p. 364-376.

\section{LEDERMANN $\mathbf{S}$.}

1969: Nouvelles tables-types de mortalité, Paris, PUF (coll. Travaux et Documents, Institut national d'études démographiques, 53).

\section{LOISON G.}

1998: «La nécropole de Pontcharaud en Basse-Auvergne", in GuILAINE J. (DIR.), Sépultures d'Occident et genèses des mégalithismes (9000-3500 avant notre ère), Séminaire du Collège de France, Paris, Errance, p. 187-206.

\section{LOISON G., VABRE V., VILLEMEUR I.}

2003: «Structures domestiques et aménagements funéraires sur le site chasséen du Crès à Béziers (Hérault)", Archéopages, 10, p. 33-39.

2004: Rocade Nord de Béziers (Hérault): Le Crès, habitats préhistoriques en bordure de l'Orb, RFO, SRA Languedoc-Roussillon, 3 vol., 429 p., 135 fig., 48 tabl.

\section{LOISON G., GLEIZE, Y.}

À paraître: «Nouvelles données sur la question des sépultures en milieu d'habitat au Chasséen ancien en Languedoc», Actes des Rencontres méridionales de Préhistoire récente de Marseille, nov. 2008.

\section{MARIÉTHOZ F.}

2007: «Variabilité des pratiques funéraires en Valais autour de 4000 av. J.-C.: découvertes récentes », in CHAMBON P., MOINAT P. (DIR.), Les Cistes de Chamblandes et la place des coffres dans les pratiques funéraires du Néolithique moyen occidental, Lausanne, Cahiers d'archéologie romande (coll. Cahiers d'archéologie romande, 110 et Mémoires de la SPF, XLIII), p. 265-276.

\section{MASSET C.}

1987: «Le "recrutement" d'un ensemble funéraire», in DUDAY H., MASSET C. (DIR.), Anthropologie physique et archéologie: méthodes d'études des sépultures, Actes du colloque de Toulouse, 4-6 nov. 1982, Paris, éd. du CNRS, p. 111-134.

Moorrees C. F. A., FANNING A., HUNT E. E.

1963a: «Age variation of formation stages for ten permanent teeth", Journal of Dental Research, 42, p. 1490-1502.

1963b: "Formation and resorption of three deciduous teeth in children", American Journal of Physical Anthropology, 21, p. 205-213.

Murail P., Bruzek J., Houët F., CUNHA E.

2005: «DSP: un outil de diagnose sexuelle probabiliste à partir des données métriques de l'os coxal», Bulletins et Mémoires de la Société d'anthropologie de Paris, 17, p. 167-176.

\section{Olivier G., Pineau H.}

1958: «Détermination de l'âge du foetus et de l'embryon ", Archives d'anatomie pathologique (coll. Suppl. à La semaine des hôpitaux de Paris), 6, p. 21-28.

PARIAT J. G.

2007: Des morts sans tombe: le cas des ossements humains en contexte non sépulcral en Europe tempérée entre le $6^{e}$ et le $3^{e}$ millénaire av. J.-C., Oxford, Archaeopress (coll. BAR International Series, 1683), 195 p.

SAUNDERS S. R.

1989: «Nonmetric skeletal variation", in ISCAN M. Y., KENNEDY K. A. R. (DIR.), Reconstruction of Life from the Skeleton, New-York, Wiley-Liss, p. 95-108. 
2000: "Subadult Skeletons and GrowthRelated Studies ", in KATZENBERG M. A., SAUNDERS S. R. (DIR.), Biological Anthropology of the human Skeleton, New York, Wiley, p. 135-161.

\section{SCHEUER L., BLACK S.}

2000: "Development and ageing of the juvenile skeleton", in COX M., MAYS S. (DIR.), Human Osteology in Archaeology and Forensic Science, Londres, Greenwich Medical Media, p. 9-22.

\section{SCHMitT A.}

2005: «Une nouvelle méthode pour estimer l'âge au décès des adultes à partir de la surface sacro-pelvienne iliaque», Bulletins et Mémoires de la Société d'anthropologie de Paris, 17, p. 89-101.

2008a: «Une nouvelle méthode pour estimer l'âge des individus décédés avant et après 40 ans ", Journal de médecine légale et de droit médical, 5, p. 17-24.

2008b: «La population chasséenne du gisement du Crès (Hérault) : caractéristiques métriques et anatomiques », Bulletins et Mémoires de la Société d'anthropologie de Paris, 20, 1-2, p. 105-124.

\section{SCOTT G. R., TURNER C. G.}

1997: The anthropology of modern human teeth: dental morphology and its variation in recent human populations, Cambridge, Cambridge University Press (coll. Cambridge studies in biological anthropology, 20), $382 \mathrm{p}$.

\section{SELlier P.}

1993: «L'estimation de l'âge des foetus et des enfants morts en période périnatale: une révision de Fazekas et Kósa (1978) », Communication au colloque Croissance et vieillissement, $21^{e}$ colloque du Groupement des anthropologistes de langue française, Bordeaux, 13-15 mai 1993, non publié, résumé des communications, p. 45.

1996: «La mise en évidence d'anomalies démographiques et leur interprétation: population, recrutement et pratiques funéraires du tumulus de Courtesoult» in PININGRE J. F. (DIR.), Nécropoles et société au premier âge du Fer: le tumulus de Courtesoult (Haute-Saône), Paris, éd. de MSH (coll. DAF, 24), p. 188-202.

\section{Testart A.}

2004: La Servitude volontaire: 1, les morts d'accompagnement, Paris, Errance, 263 p.

\section{VAQUER J.}

1998: «Les sépultures du Néolithique moyen en France méditerranéenne», in GuILAIne J. (DIR.), Sépultures d'Occident et genèses des mégalithismes (9000-3500 avant notre ère), Séminaire du Collège de France, Paris, Errance, p. 167-188.

\section{VAQUER J., DUDAY H.}

2003: «Les sépultures chasséennes du site des Plots, Berriac (Aude) ", in CHAMBON P., LECLERC J. (DIR.), Les Pratiques funéraires néolithiques avant 3500 av. J.-C. en France et dans les régions limitrophes, Paris, SPF (coll. Mémoires de la SPF, XXXIII), p. 73-79.

Vaquer J., Gandelin M., Marlière P., TEXIER M.

2008: «Les sépultures de Villeneuve-Tolosane et de Cugnaux: apports à la connaissance des pratiques funéraires du Chasséen garonnais », in VAQUER J., GANDELIN M., REMICOURT M., TCHÉRÉMISSINOFF Y. (DIR.), Défunts néolithiques en Toulousain, Toulouse, Archives d'écologie préhistorique, p. 155-178.

\section{VIGNAUD A.}

1998: «La nécropole néolithique du Camp del Ginèbre de Caramany (PyrénéesOrientales), in GUILAINE J., VAQUeR J. (DIR.), Tombes, nécropoles, rites funéraires préhistoriques et historiques, Toulouse, École des Hautes Études en Sciences Sociales (coll. Séminaires du Centre d'anthropologie), p. 19-30. 